\title{
Numerical investigation of a jet from a blunt body opposing a supersonic flow
}

\author{
Li-Wei Chen ${ }^{1}$, Guo-Lei Wang ${ }^{1}$ and Xi-Yun Lu ${ }^{1,2 \dagger}$ \\ ${ }^{1}$ Department of Modern Mechanics, University of Science and Technology of China, Hefei, \\ Anhui 230026, China \\ ${ }^{2}$ Modern Mechanics Division, E-Institutes of Shanghai Universities, Shanghai 200072, China \\ (Received 4 September 2010; revised 20 May 2011; accepted 23 June 2011; \\ first published online 30 August 2011)
}

Numerical investigation of a sonic jet from a blunt body opposing a supersonic flow with a free stream Mach number $M_{\infty}=2.5$ was carried out using large-eddy simulation for two total pressure ratios of the jet to the free stream, i.e. $\mathscr{P}=0.816$ and 1.633. Results have been validated carefully against experimental data. Various fundamental mechanisms dictating the flow phenomena, including shock/jet interaction, shock/shear-layer interaction, turbulent shear-layer evolution and coherent structures, have been studied systematically. Based on the analysis of the flow structures and features, two typical flow states, i.e. unstable and stable states corresponding to the two values of $\mathscr{P}$, are identified and the behaviours relevant to the flow states are discussed. Small-scale vortical structures mainly occur in the jet column, and largescale vortices develop gradually in a recirculation region when the jet terminates through a Mach disk and reverses its orientation as a conical free shear layer. The turbulent fluctuations are enhanced by the rapid deviation of the shear layer and the interaction with shock waves. Moreover, the coherent structures of the flow motion are analysed using the proper orthogonal decomposition technique. It is found that the dominant mode in the cross-section plane exhibits an antisymmetric character for the unstable state and an axisymmetric one for the stable state, while statistical analysis of unsteady loads indicates that the side loads can be seen as a rotating vector uniformly distributed in the azimuthal direction. Further, we clarify a feedback mechanism whereby the unsteady motion is sustained by the upstream-propagating disturbance to the Mach disk through the recirculation subsonic region and downstream propagation in the conical shear layer. Feedback models are then proposed which can reasonably well predict the dominant frequencies of the two flow states. The results obtained in this study provide physical insight into the understanding of the mechanisms relevant to the opposing jet/supersonic flow interaction.

Key words: compressible turbulence, jets, turbulence simulation

\section{Introduction}

The opposing jet in supersonic flows has attracted much attention in recent decades because of the extensive applications and the physical complexity (e.g. Warren 1950;

$\dagger$ Email address for correspondence: xlu@ustc.edu.cn 
Finley 1966; Fujita 1995; Fujita \& Karashima 1999; Shang et al. 2001; Debiève, Ardissone \& Dussauge 2003). These researchers mainly focused on improving the aerodynamic performance of aerospace vehicles, such as drag reduction and heating reduction. Moreover, the opposing jet in supersonic flows involves some important and complicated phenomena related to the coupled shearing and compressing processes, such as shock/jet interaction, shock/turbulence interaction, compressible turbulent shear-layer evolution and jet impingement (e.g. Finley 1966; Rockwell \& Naudasher 1979; Shang et al. 2001). However, the physical mechanisms dictating these phenomena are still unclear and are of great interest for further detailed studies.

The early experiments on a jet from a blunt body opposing supersonic flows mainly investigated the mean flow quantities, such as the pressure distribution on the body surface, the bow shock stand-off mean position and the shock structures (e.g. Warren 1950; Finley 1966; Karashima \& Sato 1975). These studies revealed that the total pressure ratio of the jet to the free stream is a key parameter governing the aerodynamic features. In addition, complex sustained motions of the flow field were observed experimentally in some jet conditions (e.g. Fujita \& Karashima 1999; Shang et al. 2001; Fujita 2002; Shang 2002). For example, a sonic jet from a hemispherical nose opposing a supersonic flow for a free stream Mach number $M_{\infty}=2.5$ was experimentally studied for two typical values of the total pressure or stagnation pressure ratio of the jet to the free stream (i.e. 0.816 and 1.633), and the corresponding dominant frequencies of the sustained flow motion were obtained as $3 \mathrm{kHz}$ and $17 \mathrm{kHz}$, respectively (Karashima \& Sato 1975; Fujita \& Karashima 1999; Fujita 2002). Moreover, experimental measurements and visualizations of shock motion and turbulence in a jet from a sphere against a supersonic flow $M_{\infty}=2.29$ have been taken by Debiève et al. (2003). They noticed that strong pressure fluctuations occur on the body surface and turbulent fluctuations have a significant influence on the flow characteristics.

Some numerical simulations of an opposing jet in supersonic flows have been performed to deal with the unsteady flow behaviours. The unsteady flow field around a hemispherical nose with an opposing sonic jet in a supersonic flow $M_{\infty}=2.5$ was simulated using the axisymmetric Navier-Stokes equations (Fujita 1995) and the three-dimensional (3D) equations (Fujita 2002). In these simulations, no turbulence model was used and the influence of turbulent fluctuations could not be considered. Also, a joint experimental and computational investigation was performed to study the complex self-sustained flow motion (Shang et al. 2001). They compared the computational results obtained using the Reynolds-averaged Navier-Stokes equations with turbulent closure and laminar flow equations, and found that the flow structures from the laminar computation are not supported by the experimental observations, consistent with the experimental findings by Debiève et al. (2003). Thus, these investigations indicate that turbulent simulations are needed in order to predict the flow characteristics reliably.

The sustained motions of the flow field mentioned above are associated with the shear-layer impingement phenomenon (e.g. Rockwell \& Naudasher 1979; Shang et al. 2001). A comprehensive review has indicated that the self-sustained oscillations of impinging shear layers involve a series of interacting events, such as disturbance feedback, onset of oscillation and resonance effects. The disturbance feedback is an essential feature (Rockwell \& Naudasher 1979). For a jet opposing high-speed flows, Shang et al. (2001) proposed a feedback mechanism where the oscillatory flow motions are sustained by the feedback of upstream pressure propagation through the subsonic recirculation region with a selective amplification of fluctuations in the shear 
layer. This sustained motion phenomenon is complex and requires further detailed analysis to interpret the relevant feedback mechanisms and to establish reliable feedback models.

The shock/turbulence interaction is an important issue in understanding the flow characteristics in jets opposing supersonic flows. The early linear interaction analysis on turbulence modification by a shock wave has been performed with an emphasis on acoustic wave generation behind the shock wave (Ribner 1954). Through the linear analysis, it is found that turbulent fluctuations are amplified across the shock wave and significant acoustic noise is also generated due to the interaction of vortical turbulence with the shock wave. Idealized shock/turbulence interactions have been reviewed by Andreopoulos, Agui \& Briassulis (2000). These investigations indicate that turbulence amplification through shock wave interaction is a direct effect of the Rankine-Hugoniot relations. Moreover, the flow considered here involves complex interaction phenomena and the relevant flow behaviours deserve study.

The evolution of the conical shear layer generated by the jet reversing its orientation because of the counterflow interaction has a significant influence on the turbulence structure of the flow field (Debiève et al. 2003; Chen, Xu \& Lu 2010a). Moreover, the shearing and compressing processes should be coupled to affect the shear-layer evolution. The understanding of free-shear flows is of primary interest due to the complex physics features involved. For axisymmetric compressible shear layers, some studies (e.g. Freund, Lele \& Moin 2000; Simon et al. 2007) have dealt with the 3D shape of the turbulent eddies. It is also noted that axisymmetric compressible shear layers may lead to the occurrence of a wide range of phenomena coupled with complex interactions (Simon et al. 2007). In the flow considered here, the rapid deviation of the shear layer occurs as the jet terminates through a Mach disk, and then the shear-layer reattachment to the body causes a pressure rise (e.g. Finley 1966; Shang et al. 2001). Thus, both the streamline curvature and the adverse pressure gradient along the conical shear layer constitute extra strain rates as defined by Bradshaw (1974), which will have an important influence on the turbulent flow field.

The unsteady flow field of a jet from a blunt body opposing supersonic flows involves an array of intricate phenomena, such as shock/jet interaction, shock/turbulence interaction, recirculation zone, and cell structures in the jet column. Characterizing dynamical processes in flow evolution is still one of the fundamental challenges in fluid mechanics. Usually, the dynamical processes are analysed by the proper orthogonal decomposition approach (Lumley 1967; Berkooz, Holmes \& Lumley 1993) and vortical structures are often discussed relative to the importance of vortices (e.g. Robinson 1991; Jeong \& Hussain 1995). As a typical example, Meyer, Pedersen \& Özcan (2007) employed the proper orthogonal decomposition to investigate a turbulent jet in crossflow and to analyse the coherent vortical structures and the interactions between the jet core and the crossflow.

In this paper, a large-eddy simulation (LES) technique, which has provided a powerful tool for studying the dynamics of turbulent flows, is utilized to investigate the complicated interactions between a jet issued from a blunt body and a supersonic counterflow. The purpose is to achieve an improved understanding of some of the fundamental phenomena involved in this flow, including shock/jet interaction, shock/shear-layer interaction, turbulent shear-layer evolution and coherent structures.

This paper is organized as follows. The mathematical formulation and numerical method are briefly presented in $\S 2$. The computational overview and validation are described in $\S 3$. Detailed results are then given in $\S 4$ and concluding remarks in $\S 5$. 


\section{Mathematical formulation and numerical methods}

\subsection{Governing equations and turbulence modelling}

To investigate a jet from a blunt body opposing a supersonic flow, the threedimensional Favre-filtered compressible Navier-Stokes equations in generalized coordinates are employed. The equation of state for an ideal gas is used and the molecular viscosity is assumed to obey the Sutherland law. To non-dimensionalize the equations, we use the free stream variables including the density $\rho_{\infty}$, temperature $T_{\infty}$, speed of sound $a_{\infty}$, and the diameter of the hemisphere $D$ as characteristic scales.

The LES is implemented in the present work for turbulence closure. Some terms in the Favre-filtered equations arise from unresolved scales and need to be modelled in terms of resolved scales. Then, dynamic subgrid-scale (SGS) models for compressible flows are employed. A detailed description of the mathematical formulation of the nondimensionalized equations and the subgrid-scale models can be found in our previous papers (Lu et al. 2005; Xu, Chen \& Lu 2010).

\subsection{Numerical procedure}

The governing equations are numerically solved by a finite-volume method. The convective terms are discretized by a central/upwind hybrid scheme for shockcapturing and the viscous terms by a fourth-order central difference (Xu et al. 2010). The temporal integration is performed using an implicit approximate-factorization method with sub-iterations to ensure second-order accuracy (Simon et al. 2007).

To capture the discontinuity caused by a shock wave, a second-order upwind scheme with Roe's flux-difference splitting is introduced into the inviscid flux. Based on the detection of shocks proposed by Hill, Pantano \& Pullin (2006), the spatial discretization has been constructed explicitly to be shock capturing with the upwind scheme and to revert to a central stencil with a fourth-order central scheme (Rai \& Chakravarthy 1993; Génin \& Menon 2010) in turbulent flow regions away from shock. A detailed description of the formulation can be found in our previous papers (Chen, Xu \& Lu 2010b; Xu et al. 2010).

In the present study, the initial and boundary conditions are given as follows. The initial condition is set as the free-stream quantities. No-slip and adiabatic conditions are applied on the body surface. The far-field boundary and downstream boundary conditions are treated by a characteristic method based on Riemann invariants (Thomas \& Salas 1986). The jet boundary conditions are set on the surface using a specified velocity profile, total temperature and constant static pressure (e.g. Bodony \& Lele 2005; Bogey \& Bailly 2006). The density can be obtained by using the Crocco-Busemann relation. The jet velocity profile is chosen as the hyperbolic-tangent function (Bodony \& Lele 2005)

$$
\frac{\bar{u}}{U_{j}}=\frac{1}{2}\left(1-\tanh \left[\frac{1}{4 \theta_{0}}\left\{\frac{r}{r_{0}}-\frac{r_{0}}{r}\right\}\right]\right),
$$

where $U_{j}$ is the velocity at the jet axis, $\theta_{0}$ is the initial momentum thickness, $r_{0}$ is the jet radius with the diameter $D_{j}=2 r_{0}$, and $r=\sqrt{y^{2}+z^{2}}$. As suggested by Bodony $\&$ Lele (2005), $\theta_{0} / D_{j}=0.045$ in the calculations. Small random disturbances are also added to the mean velocity profiles to seed the turbulence development (Bogey \& Bailly 2006). 


$\begin{array}{lcccc} & \text { Grid } & a_{\infty} \Delta t / D & S t(\mathscr{P}=0.816) & S t(\mathscr{P}=1.633) \\ \text { Case 1 } & 150 \times 150 \times 80 & 0.005 & 0.231 & 1.453 \\ \text { Case 2 } & 200 \times 200 \times 120 & 0.002 & 0.251 & 1.497 \\ \text { Case 3 } & 300 \times 300 \times 240 & 0.001 & 0.252 & 1.506 \\ \text { EXP } & & & 0.262 & 1.485\end{array}$

TABLE 1. Validation of grid resolution and time step. Here, $S t=f D / U_{\infty}$ with $f$ being the dominant frequency of the sustained flow motion and $D$ the diameter of the hemisphere; EXP represents the experimental data with $f=3 \mathrm{kHz}$ at $\mathscr{P}=0.816$ and $f=17 \mathrm{kHz}$ at $\mathscr{P}=1.633$ (Fujita 2002).

\section{Computational overview and validation}

\subsection{Computational overview}

We consider a supersonic flow with a free-stream Mach number $M_{\infty}=2.5$ around a hemispherical nose perturbed by an opposing sonic jet placed on its axis. Based on previous experiments (e.g. Karashima \& Sato 1975; Fujita \& Karashima 1999; Fujita 2002), an ideal gas is assumed for the free stream and the jet. The diameter of the injector $D_{j}$ is set as $D / 10$. The values of the total pressure or stagnation pressure ratio of the jet to the free stream $\mathscr{P}=p_{0 j} / p_{0 \infty}$ are chosen as 0.816 and 1.633, and the corresponding momentum flux ratios $J=\rho_{j} U_{j}^{2} / \rho_{\infty} U_{\infty}^{2}$ are obtained as 1.178 and 2.358. The total temperature or stagnation temperature ratio $\mathscr{T}=T_{0 j} / T_{0 \infty}$ is 1.0 with $T_{0 \infty}=294 \mathrm{~K}$. The Reynolds number defined as $R e_{D}=U_{\infty} D / \mu_{\infty}$ is $1.47 \times 10^{6}$. The selected parameters are the same as those in the experiments. The relevant experimental data will thus be employed to validate the present calculation.

The grid topology is carefully designed to ensure the computation accuracy. An orthogonal grid distribution in the meridian plane is used and the azimuthal grid is divided uniformly. Grid stretching is employed to increase the grid resolution near the body surface and in the nose region and ensures that there are at least 55 nodes located in the radius of the injector. The minimum size of the grid in the wall-normal direction is $\Delta y_{n}=5 \times 10^{-5} D$, corresponding to $\Delta y_{n}^{+}<0.1$ based on the wall friction parameters. The tangential spacing on the surface of the spherical nose lies in the range $\Delta s^{+} \sim 12-16$, which is fine enough to capture the fine-scale flow structures (e.g. Georgiadis, Rizzetta, Fureby 2010).

To assess the effects of grid resolution and time step on the calculated results, three typical cases with different grid resolutions and time steps are listed in table 1. Detailed comparisons of the calculated results using different computational conditions will be given in $\S 3.2$. The present code is equipped with a multi-block domain decomposition feature to facilitate parallel computing and the computational domain is divided into 32 sub-domains for parallel processing. The computation runs for time $\sim 200 D / U_{\infty}$ to obtain statistically meaningful turbulence properties in the temporal averaging operation.

Several averaging operations in the post-processing are performed based on the timedependent resolved density $\bar{\rho}$, pressure $\bar{p}$, temperature $\tilde{T}$ and velocity $\tilde{u}_{i}$. Moreover, the velocity $\tilde{u}_{i}$ is represented in the cylindrical coordinate system and the corresponding axial, radial and azimuthal components are denoted $\tilde{u}_{x}, \tilde{u}_{r}$ and $\tilde{u}_{\phi}$. To clearly present the post-processing, some symbols used in this paper are introduced as follows: \langle\rangle means the average in time (after careful elimination of the transient part of the 

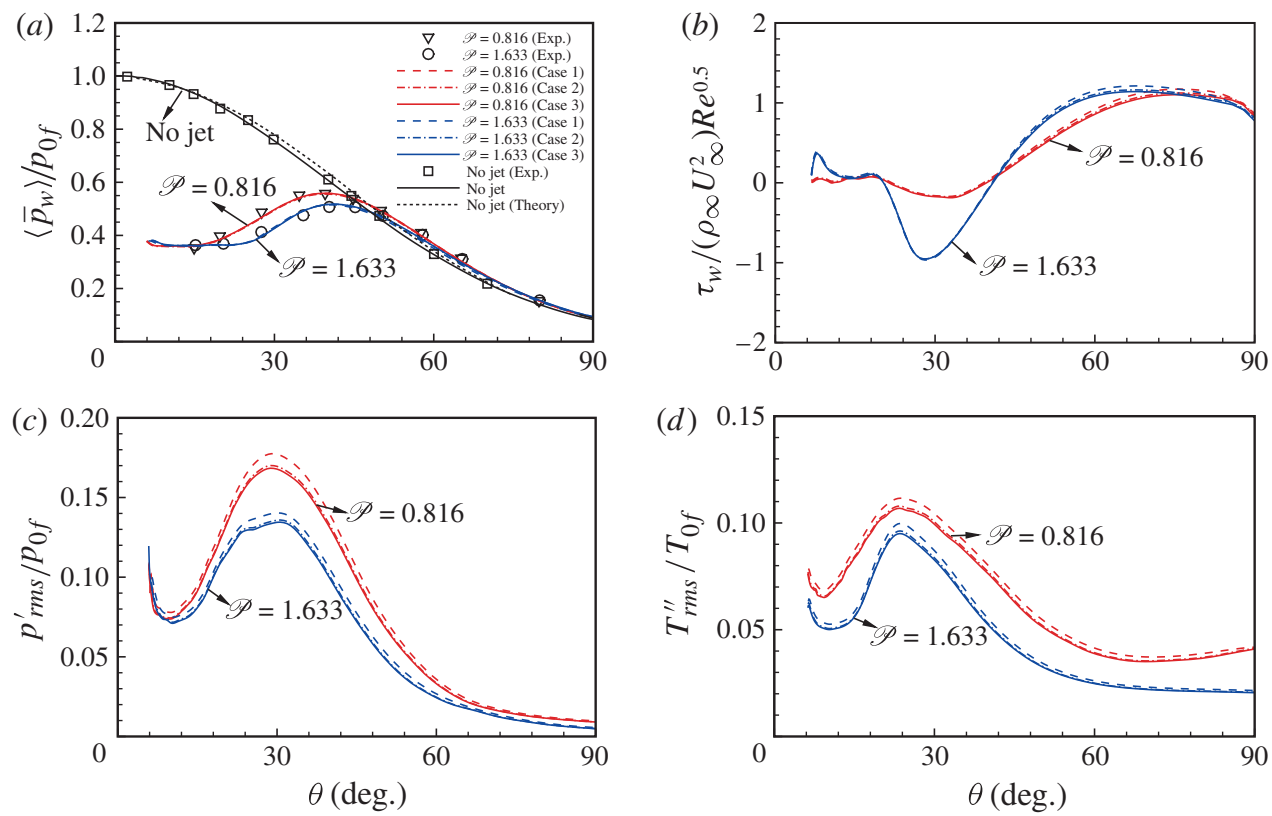

FIGURE 1. (Colour online available at journals.cambridge.org/flm) Comparison of the calculated results with experimental data and validation of the results obtained by different grid resolutions and time steps (i.e. cases $1-3$ ) given in table 1 for $\mathscr{P}=0.816$ and 1.633: (a) mean pressure $\left\langle\bar{p}_{w}\right\rangle$ and experimental data (Karashima \& Sato 1975; Fujita \& Karashima 1999), (b) mean skin-friction coefficient, $(c)$ pressure fluctuation $p_{r m s}^{\prime}$, and $(d)$ temperature fluctuation $T_{r m s}^{\prime \prime}$ on the body surface. Here, $\theta$ increases from the axisymmetric axis to the shoulder of the blunt body. In $(a)$, the axisymmetric result without an opposing jet obtained with grid $300 \times 300$ and comparisons with the experimental data at $M_{\infty}=2.5$ and $R e_{D}=1.4 \times 10^{6}$ (Finley 1966) and modified Newtonian theory (Finley 1966) are also shown.

time-dependent variations) and in the azimuthal direction, and $\{f\}=\langle\bar{\rho} f\rangle /\langle\bar{\rho}\rangle$ for a variable $f$. Then, the fluctuations are obtained as (Garnier, Sagaut \& Deville 2002) $\rho^{\prime}=\bar{\rho}-\langle\bar{\rho}\rangle, p^{\prime}=\bar{p}-\langle\bar{p}\rangle, T^{\prime \prime}=\tilde{T}-\{\tilde{T}\}$ and $u_{i}^{\prime \prime}=\tilde{u}_{i}-\left\{\tilde{u}_{i}\right\}$, respectively.

\subsection{Validation}

To validate the present simulation, we compare numerical results and experimental data (Finley 1966; Karashima \& Sato 1975; Fujita \& Karashima 1999) in terms of the mean wall pressure and the dominant frequency of flow motion as given in table 1 . Figure 1(a) shows the distributions of mean wall pressure $\left\langle\bar{p}_{w}\right\rangle / p_{0 f}$, where $p_{0 f}$ is the stagnation pressure downstream of the bow shock. The results compare well with the experimental data. Moreover, validation of the results predicted by different grid resolutions and time steps (i.e. cases 1-3) listed in table 1 is also performed. It is shown that the results for the three cases are consistent with the experimental data.

Assessments of the effect of grid resolution and time step on the calculated solutions have been performed. Figure $1(b-d)$ shows the distributions of the skinfriction coefficient $\tau_{w}$, and the pressure and temperature fluctuations on the surface, respectively. We have identified that the results for cases 2 and 3 collapse together, indicating a reasonable convergence for the grid resolution and time step given in table 1. Moreover, an axisymmetric result without an opposing jet was obtained 
with grid $300 \times 300$, which agrees well with the experimental data (Finley 1966) and modified Newtonian theory (Finley 1966). To make the prediction accurate, the results given below were calculated using the parameters in case 3 , i.e. the number of grid points $300 \times 300 \times 240$ in the axial $(x)$, radial $(r)$ and azimuthal $(\phi)$ direction, respectively, and time step $0.001 D / a_{\infty}$.

The resolved energy spectrum obtained using the parameters in case 3 has been examined. The resolved scales seem to reach an inertial subrange, reasonably close to $S t^{-5 / 3}$ scaling (Kawai \& Fujii 2005). The spatial spectrum can be approximately obtained using Taylor's hypothesis which is limited to homogeneous turbulence with small turbulence intensity (Pope 2000). Moreover, the resolved energy spectra obtained by the present numerical method were also examined in some previous investigations (e.g. Chen et al. 2010b; Xu et al. 2010).

We have further assessed the appropriateness of the grid resolution used in this study in terms of the SGS turbulent kinetic energy (SGS-TKE) and the Kolmogorov scale. Similar to the treatment in Matheou et al. (2010), we have calculated the SGS-TKE using the data in case 3 and identified that the ratio of the SGS-TKE to the total TKE is less than $20 \%$, indicating a sufficiently resolved LES resolution (Pope 2004; Ferrante, Matheou \& Dimotakis 2011). Moreover, the Kolmogorov scale $\eta$ is also estimated as $\eta / D \sim O\left(10^{-4}\right)$ and the grid spacing in the shear layer region is $\delta / D \sim O\left(10^{-3}\right)$. Then, the ratio $\delta / \eta$ is $O(10)$. Compared to the LES of a mixing layer with $\delta / \eta \sim O\left(10^{2}\right.$ ) (Matheou et al. 2010), it is verified that the grid resolution is sufficient to resolve turbulence in the flow field. Thus, the quality of the present simulation is capable of capturing meaningful turbulence.

Furthermore, the present numerical strategy has already been applied with success to a wide range of turbulent flows such as compressible turbulent swirling flows injected into a coaxial dump chamber (Lu et al. 2005) as well as compressible flows past an aerofoil (Chen et al. 2010b) and over a circular and wavy cylinder (Xu, Chen \& Lu 2009; $\mathrm{Xu}$ et al. 2010). We have carefully examined the physical model and numerical approach used in this study and have verified that the calculated results are reliable.

\section{Results and discussion}

\subsection{Flow structures and flow features}

\subsubsection{Mean flow structures}

The mean flow quantities are obtained in terms of the time average and spatial average in the azimuthal direction. The numerical schlieren pattern and streamlines of the mean flow for $\mathscr{P}=0.816$ and 1.633 are shown in figure $2(a, b)$, respectively. As a jet issues from the body against the supersonic flow, the bow shock stands away from the body surface and takes a form appropriate for a combined influence of the body and the jet flow. From the numerical schlieren pattern in figure 2(a), the structure within the jet exhibits two oblique shocks with regular reflection to form an X-type structure in the plane (or a conical shock wave structure in 3D) and the jet column terminates at a Mach disk, across which the jet total pressure is reduced to the free stagnation value. The fluid from the jet reverses its orientation as a conical free shear layer and is partly entrained to generate a recirculation zone outside the jet column, which is clearly depicted by the streamlines. As the conical shear layer reattaches to the body surface, the flow realigns with the body contour and also causes a pressure rise; a reattachment shock is then generated which intersects with the bow shock to form a triple point. Because of the unsteady motion of the shock system shown below, the shocks are blurred based on the mean pattern in figure $2(a)$, consistent with the 
(a)

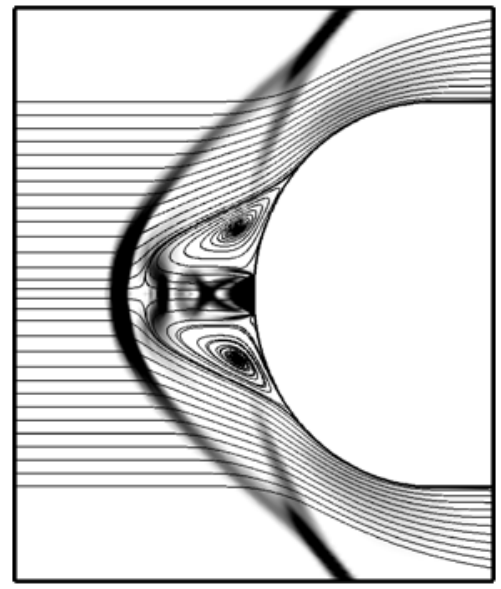

(b)

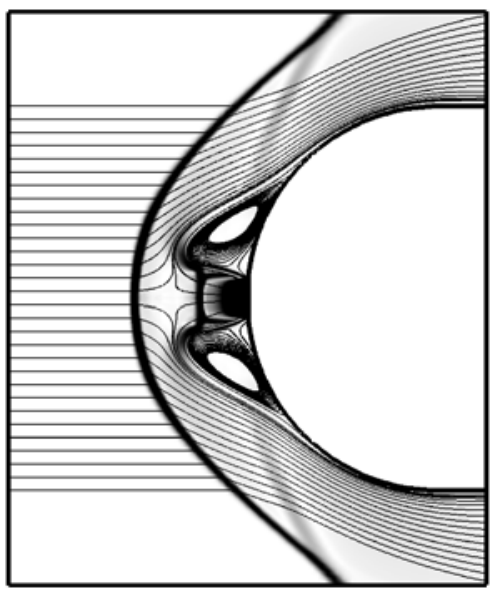

FIGURE 2. Numerical schlieren-like visualization by contours of $\|\nabla \bar{\rho}\|$ and streamlines of the mean flow for $(a) \mathscr{P}=0.816$ and $(b) 1.633$.

experimental photographs (Karashima \& Sato 1975; Shang et al. 2001). Moreover, the phenomena described above can be confirmed based on the temporal evolution of the flow field and will be further discussed later.

For $\mathscr{P}=1.633$ in figure $2(b)$, the jet exhibits a single-cell structure with a well established Mach disk at the end of the jet column and a barrel shock inside the jet layer. The other features of the mean flow field are similar to those in figure 2(a), such as the recirculation zone outside the jet column, the reattachment shock and its intersection with the bow shock. The relevant difference of the mean flow structures in figures $2(a)$ and is $2(b)$ associated with two typical flow states, i.e. unstable state for $\mathscr{P}=0.816$ and stable state for $\mathscr{P}=1.633$ (Karashima \& Sato 1975; Fujita 1995; Fujita \& Karashima 1999). Moreover, the bow shock stand-off mean position is obtained as $x / D=-0.849$ for $\mathscr{P}=0.816$ and $x / D=-0.792$ for $\mathscr{P}=1.633$, which agree well with the experimental data -0.85 and -0.789 (Karashima \& Sato 1975; Fujita \& Karashima 1999), respectively.

The existence of shock/turbulence interaction is related to the compressible effect. To understand this flow feature, figure 3 shows the local Mach number based on the mean flow quantities in the meridian plane. The distributions of local Mach number clearly distinguish the supersonic and subsonic regions. From figure 3(a), the subsonic region exists behind the core region of the bow shock and in the recirculation zone and the conical shear-layer region. The supersonic flow occurs in the jet column and the local supersonic Mach number does not change much across the reattachment shock since it is weak. From figure $3(b)$ at $\mathscr{P}=1.633$, the subsonic region over the recirculation zone and the conical shear layer are smaller compared to figure $3(a)$ at $\mathscr{P}=0.816$, which may be related to the magnitude of the dominant frequency of this self-sustained unsteady flow system. Moreover, the disturbances possibly propagate in the subsonic region to influence the jet structure. A feedback mechanism of the free shear layer and the Mach disk was proposed by Shang et al. (2001). The relevant feedback mechanisms for the two flow states will be discussed in detail in $§ 4.3 .2$.

\subsubsection{Instantaneous flow structures}

The instantaneous flow field is further examined to analyse the flow phenomena. Figure $4(a, b)$ shows the flow structures using the iso-contours of $\|\nabla \bar{\rho}\|$ in the 

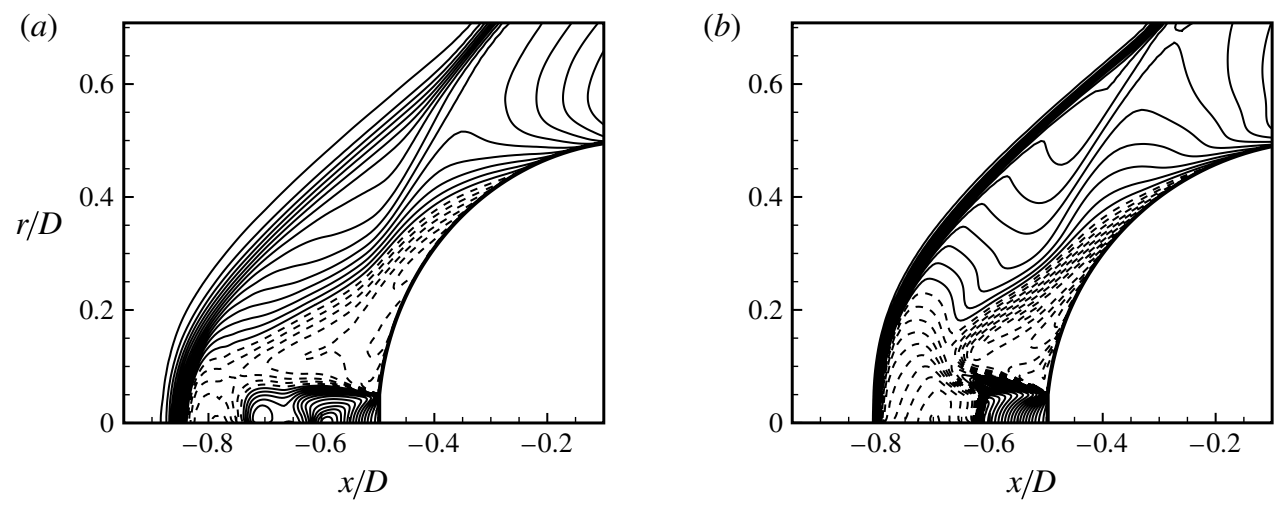

FIGURE 3. Distributions of the mean local Mach number $M_{l}$ for $(a) \mathscr{P}=0.816$ and $(b)$ 1.633. Here, solid lines denote $M_{l}>1$ and dashed lines $M_{l}<1$ with a contour increment 0.1 .

meridian planes for $\mathscr{P}=0.816$. The deviation of the jet back to the body results in a complicated conical shear layer (Finley 1966; Shang et al. 2001), as marked in figure $4(a)$. The conical shear layer eventually reattaches to the body surface, triggering a series of compression waves in the near region of body surface and causing a pressure rise, which is also confirmed by the mean pressure distribution on the body surface in figure 1(a). Then, a reattachment shock is generated and intersects with the bow shock to form a triple point in the plane. According to the evolution of the flow structures, it is identified that the bow shock surface presents a small distortion because of the disturbances from the jet and the conical shear layer. Moreover, the bow shock interacting with the shear layer results in a self-sustained off-axis motion of flow structures, which induces a blurred schlieren pattern of the mean flow field in figure 2(a). This kind of flow behaviour is usually classified as the unstable state (Karashima \& Sato 1975; Fujita 1995; Fujita \& Karashima 1999).

To clearly illustrate the flow structures in the jet, figure $4(c, d)$ shows the corresponding enlarged patterns. The jet flow undergoes a series of expansion waves and then experiences a pair of oblique shocks in the plane. The oblique shocks interact with a regular reflection to form an X-type structure, consistent with the experimental observations. Moreover, the jet layer becomes unstable quickly and the vortical coherent structures convect downstream on the outside of the jet column. Since the subsonic region occurs as typically shown in figure $3(a)$, there exists a feedback resonance mechanism between the conical shear layer and the Mach disk of this flow field (Shang et al. 2001). The jet structures undergo a corresponding change subsequently. As shown in figure 4(c), the jet layer evolves into the jet column behind the X-type structure and the Mach disk nearly disappears. Then, the oblique shocks become somewhat weak and the Mach disk appears again in figure $4(d)$.

Figure 5(a,b) shows the instantaneous flow structures using the iso-contours of $\|\nabla \bar{\rho}\|$ in the meridian planes for $\mathscr{P}=1.633$. As marked in figure $5(a)$, a complicated conical shear layer is generated and reattaches to the body surface. Meanwhile, a series of compression waves is triggered in the near region of the body surface and a reattachment shock is formed which intersects with the bow shock. The corresponding jet structures are shown in figure $5(c, d)$. Similar to the case of $\mathscr{P}=0.816$, as a feedback resonance mechanism occurs, the jet structures are also accompanied by the evolution of the conical shear layer. From figure 5(c), the jet cell structure exhibits a Mach reflection with a well established Mach disk and barrel shock. A reflected 
(a)

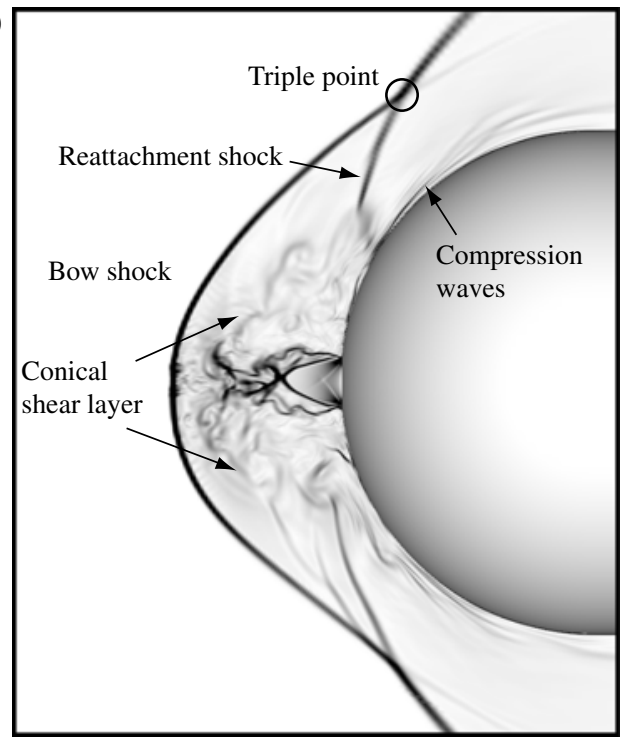

(c)

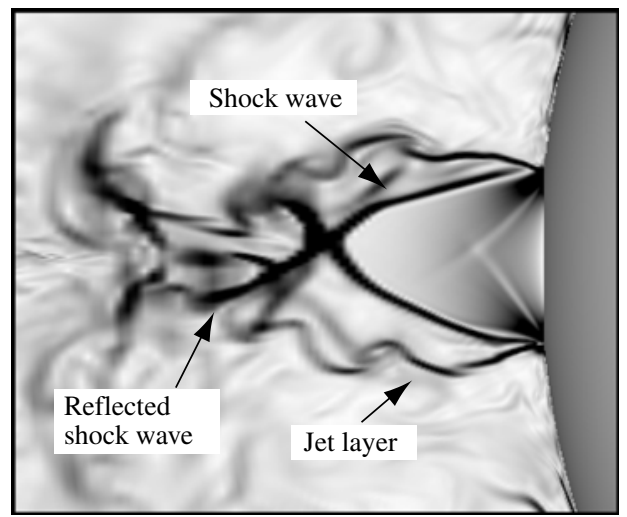

(b)

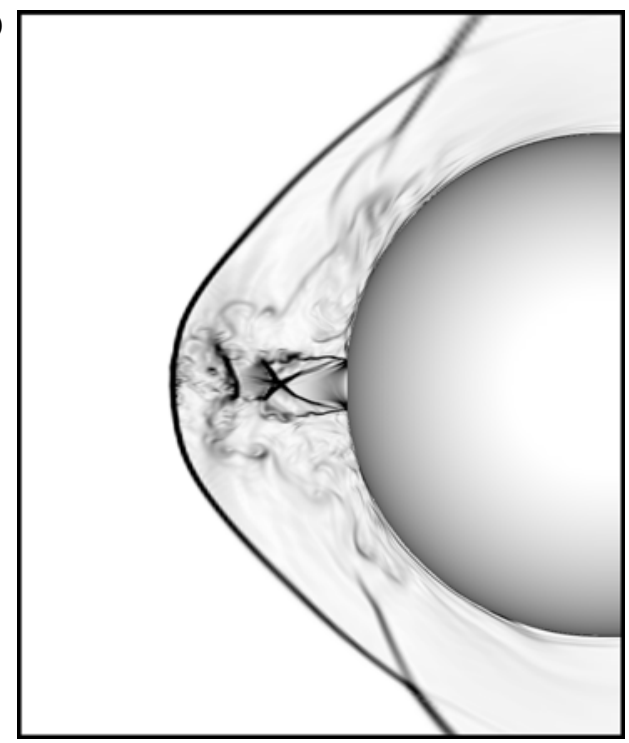

$(d)$

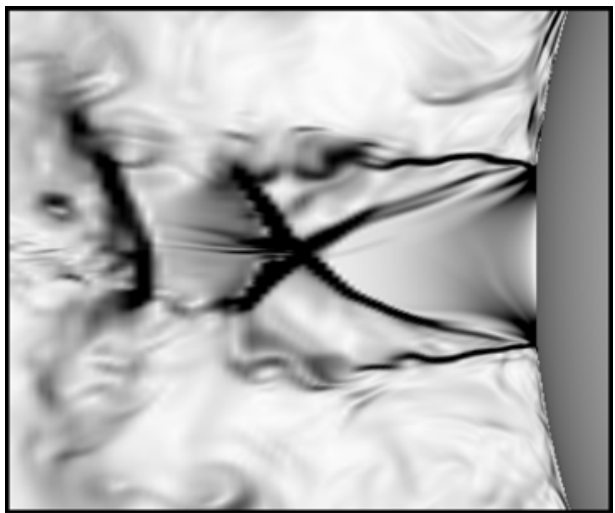

FIGURE 4. Numerical schlieren-like visualizations by contours of $\|\nabla \bar{\rho}\|$ in the meridian plane for $\mathscr{P}=0.816$ at two instants in $(a)$ and $(b)$ and the corresponding enlarged jet structures in $(c)$ and $(d)$.

shock is formed from the intersection of the barrel shock with the Mach disk. Then, from the influence of the conical shear layer, the barrel shock becomes obviously weak and the Mach disk behaves like a normal shock wave in figure $5(d)$. We have carefully examined the evolution of the flow structures and identified that the barrel shock occurs intermittently. As suggested in previous works (Karashima \& Sato 1975; Fujita 1995; Fujita \& Karashima 1999), this kind of flow is classified as the stable state. Note that there still exists unsteady motion in the stable state.

As illustrated above for the instantaneous flow structures, motion of the bow shock and reattachment shock are observed because of the perturbation of the entire flow (Debiève et al. 2003). The evolution of the jet structures is closely associated with the unsteady off-axis motion, consistent with the experimental observations (e.g. Karashima \& Sato 1975; Shang et al. 2001). These 3D flow phenomena cannot be predicted by an axisymmetric simulation (e.g. Fujita 1995; Shang 2002). Moreover, 
(a)

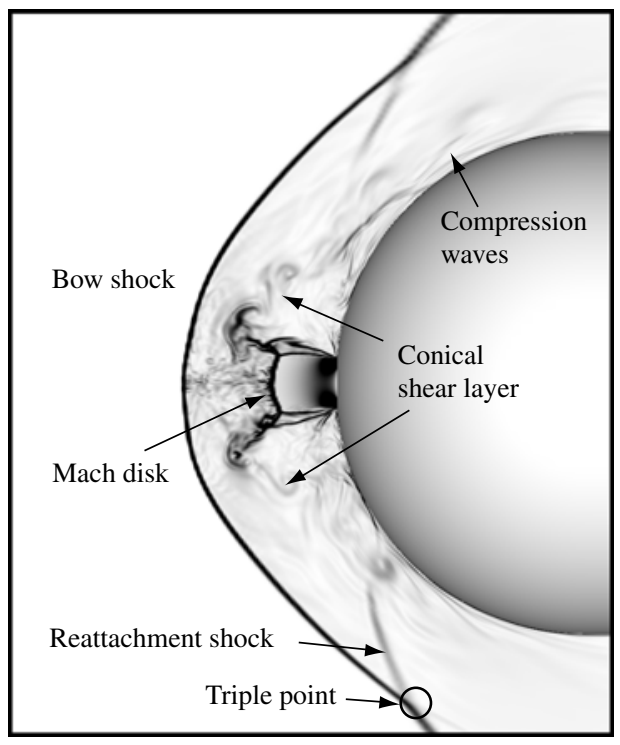

(c)

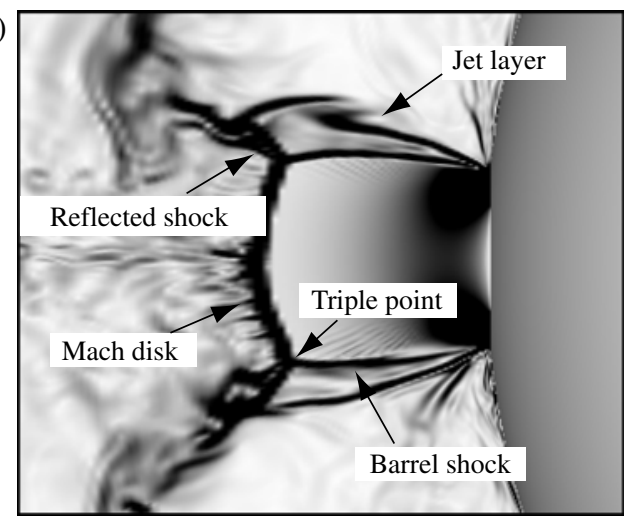

(b)

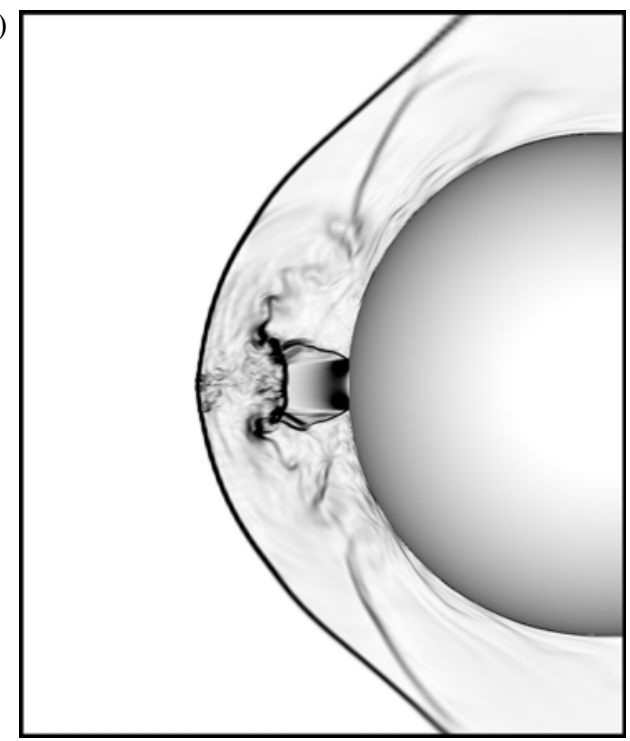

$(d)$

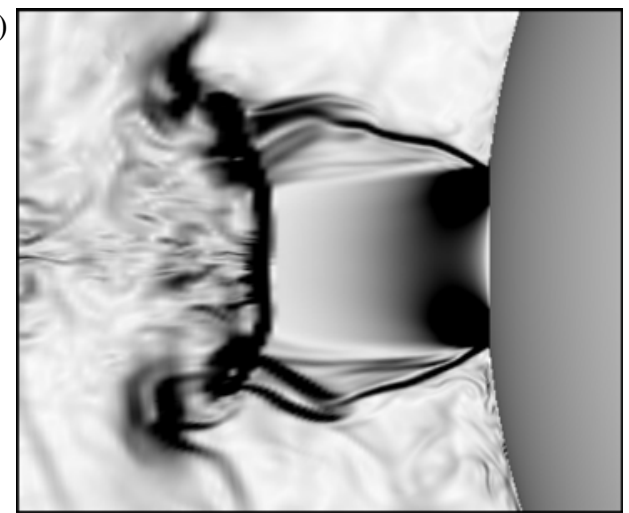

FIGURE 5. Numerical schlieren-like visualizations by contours of $\|\nabla \bar{\rho}\|$ in the meridian plane for $\mathscr{P}=1.633$ at two instants in $(a)$ and $(b)$ and the corresponding enlarged jet structures in $(c)$ and $(d)$.

according to experimental measurement of a jet issuing from a sphere against a supersonic flow (Debiève et al. 2003), the conical shear layer originating from the deviation of the jet leads to apparent turbulent fluctuations. Thus, turbulence has a significant influence on the flow characteristics and will be discussed in $\S 4.2 .1$.

To assess the existence of vortical structures in the flow field, figure 6 shows an instantaneous snapshot of the flow field depicted by an iso-surface of the $Q$ criterion (Jeong \& Hussain 1995)

$$
Q=-\frac{1}{2}\left(\|\boldsymbol{S}\|^{2}-\|\boldsymbol{\Omega}\|^{2}\right),
$$

where $\boldsymbol{S}$ and $\boldsymbol{\Omega}$ denote the strain and the rotation tensors, respectively. A positive value of $Q$ represents the regions where the rotation exceeds the strain. It must be recalled that the criterion (4.1) is only applied to the resolved scales obtained by 
(a)

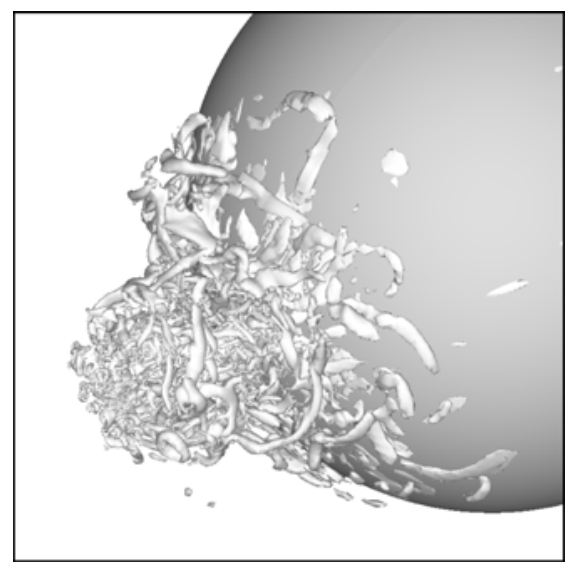

(b)

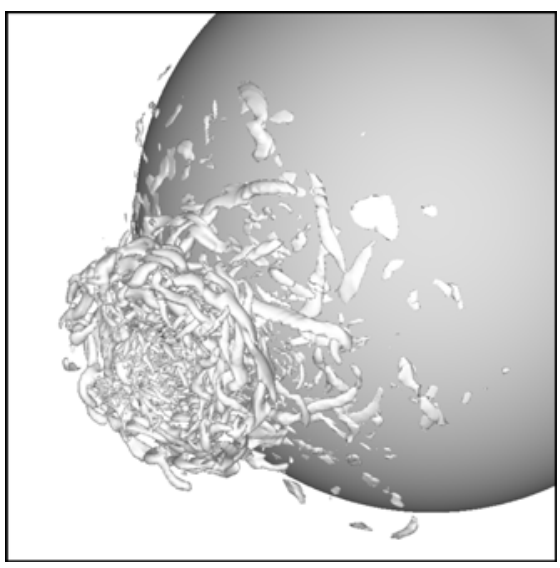

FIGURE 6. Iso-surface of the $Q$ criterion for $(a) \mathscr{P}=0.816$ and $(b) 1.633$.

LES and that the vortical structures could be different if the whole flow field were considered (Simon et al. 2007; Chen et al. 2010b).

From the region occupied by the vortical structures, it is reasonably well identified that the jet penetration at $\mathscr{P}=1.633$ is higher than at $\mathscr{P}=0.816$, consistent with the bow shock stand-off position obtained above. The small-scale vortical structures mainly occur in the jet column. When the jet terminates through the Mach disk and the fluid from the jet is deflected back, large-scale vortices develop gradually in the outer region of the jet column, corresponding to the recirculation zone (figure 2) with a subsonic flow (figure 3). The occurrence of large-scale hairpin vortices in the attachment region is clearly shown in figure 6(a). This kind of coherent structure was also observed in backward-facing step flows (Robinet 2007) and axisymmetric separating-reattaching flow (Deck \& Thorigny 2007). An apparent unsteady evolution of the conical shear layer has been identified in the unstable state, which is caused by the convection of large-scale vortices along the shear layer, leading to an obvious asymmetrical distribution of the instantaneous pressure in the azimuthal direction (Bourdon \& Dutton 2000). Moreover, as shown in figure 6(b), the rolled-up eddies exhibit a vortex-ring-like axisymmetric coherent structure convecting downstream and interact with the body surface.

\subsubsection{Dynamic features on the body surface}

The flow structures are closely associated with the dynamic features on a body (Wu, Lu \& Zhuang 2007). The mean pressure $\left\langle\bar{p}_{w}\right\rangle / p_{0 f}$ on the surface is shown in figure $1(a) ;\left\langle\bar{p}_{w}\right\rangle$ varies smoothly with $\theta$, then rises to a maximum at $\theta=38^{\circ}-40^{\circ}$ and finally decreases, tending to the value with no jet. The pressure rise is related to the reattachment of the conical shear layer as shown in figures 4 and 5. According to the analysis by Finley (1966), the reattachment is believed to occur just before the pressure maximum, which therefore can be called the reattachment pressure. Moreover, a reattachment process in steady flow is usually characterized by the local skin friction vanishing on the surface. Thus, from the mean skin friction in figure $1(b)$, it is identified that the mean reattachment point is around $\theta=40^{\circ}$, consistent with the location of the reattachment pressure.

The root-mean-square value of pressure fluctuation $p_{r m s}^{\prime} / p_{0 f}$ along the surface is also shown in figure $1(c)$. It exhibits a higher value at $\theta=5^{\circ}$ because of the influence 

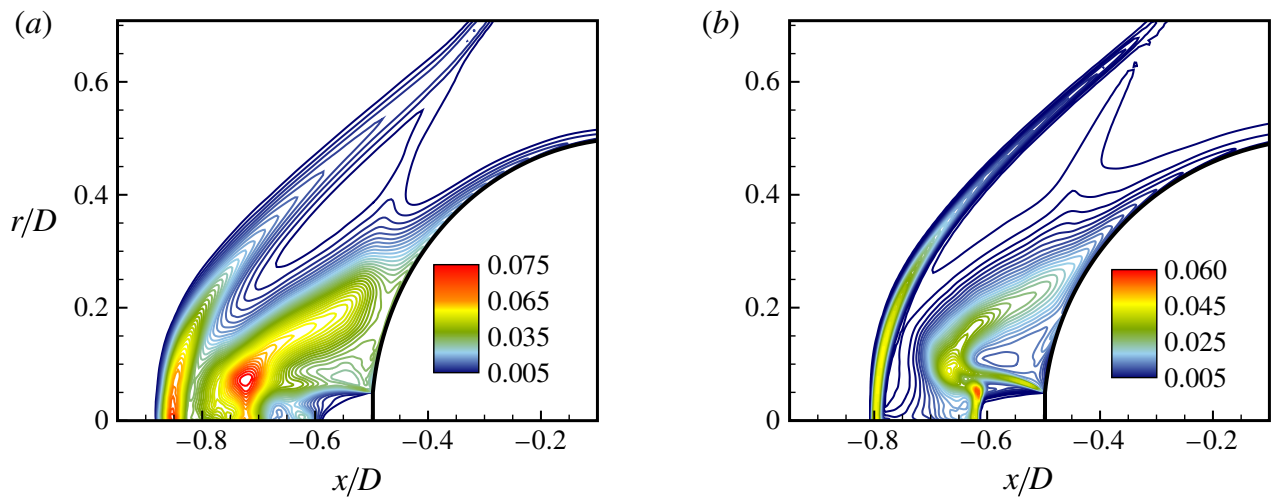

FIGURE 7. Distributions of the specific turbulent kinetic energy for $(a) \mathscr{P}=0.816$ and (b) 1.633 .

of the jet layer. As $\theta$ increases, $p_{r m s}^{\prime}$ increases quickly after a minimum at $\theta=10^{\circ}$, which is related to the fact that the organized shear-layer structures become stronger and evolve closer to the surface (Hudy, Naguib \& Humphreys 2003). Then $p_{r m s}^{\prime}$ reaches its maximum 0.17 at approximately $\theta=29^{\circ}$ for $\mathscr{P}=0.816$ and 0.14 at $\theta=32^{\circ}$ for $\mathscr{P}=1.633$, lying upstream of the mean reattachment location. Further, $p_{r m s}^{\prime}$ decreases along the surface due to the flow acceleration and the surface convex curvature (Pope 2000; Debiève et al. 2003). For comparison, the value of $p_{r m s}^{\prime} / p_{0 f}$ at $\theta=15^{\circ}$ is 0.09 for $\mathscr{P}=0.816$ and 0.08 for $\mathscr{P}=1.633$; the corresponding axisymmetric values were obtained as 0.10 and 0.09 by Fujita (1995). Moreover, as shown in figure $1(d)$ the temperature fluctuation increases from its minimum to maximum at $\sim \theta=23^{\circ}$ and then decreases gradually to an approximate equilibrium value in the attached boundary layer of $\sim 0.04$ for $\mathscr{P}=0.816$ and 0.02 for $\mathscr{P}=1.633$. Obviously, the pressure and temperature fluctuations at $\mathscr{P}=0.816$ for the unstable state are higher than those at $\mathscr{P}=1.633$ for the stable state.

\subsection{Turbulent flow field and side loads}

\subsubsection{Velocity fluctuations and conical shear-layer}

For a jet issuing from a sphere against a supersonic flow, the shearing process of the jet layer and conical shear layer can result in strong turbulent fluctuations, which will have a significant influence on the flow characteristics (Debiève et al. 2003). Moreover, the shock movement which is related to the compressing process also strengthens the flow fluctuations (Andreopoulos et al. 2000). It should be expected that the fluctuations due to shock movement show up in the turbulence statistics. Figure 7 shows typical the iso-contours of the specific turbulent kinetic energy (TKE), i.e. $k=\left\{u_{i}^{\prime \prime} u_{i}^{\prime \prime}\right\} / 2 U_{\infty}^{2}$. The distribution of higher TKE mainly occurs in the regions of the deviation of the jet and downstream of the bow shock. It is identified that its maximum value is around 0.076 for $\mathscr{P}=0.816$ and 0.063 for $\mathscr{P}=1.633$ in the region where the jet interacts with the Mach disk and is deflected back. Moreover, the region of higher TKE for $\mathscr{P}=0.816$ is larger than that for $\mathscr{P}=1.633$ because of the influence of strong unsteadiness of the unstable state.

The turbulent flow field is mainly influenced by the conical shear layer because of the deviation of the jet (Debiève et al. 2003). To understand the conical shear-layer evolution, the iso-contours of specific turbulent shear stress $\tau_{x r}^{\prime \prime}=\left\{u_{x}^{\prime \prime} u_{r}^{\prime \prime}\right\} / U_{\infty}^{2}$ are shown in figure $8(a, b)$ for $\mathscr{P}=0.816$ and 1.633 , respectively. Positive $\tau_{x r}^{\prime \prime}$ mainly occurs 

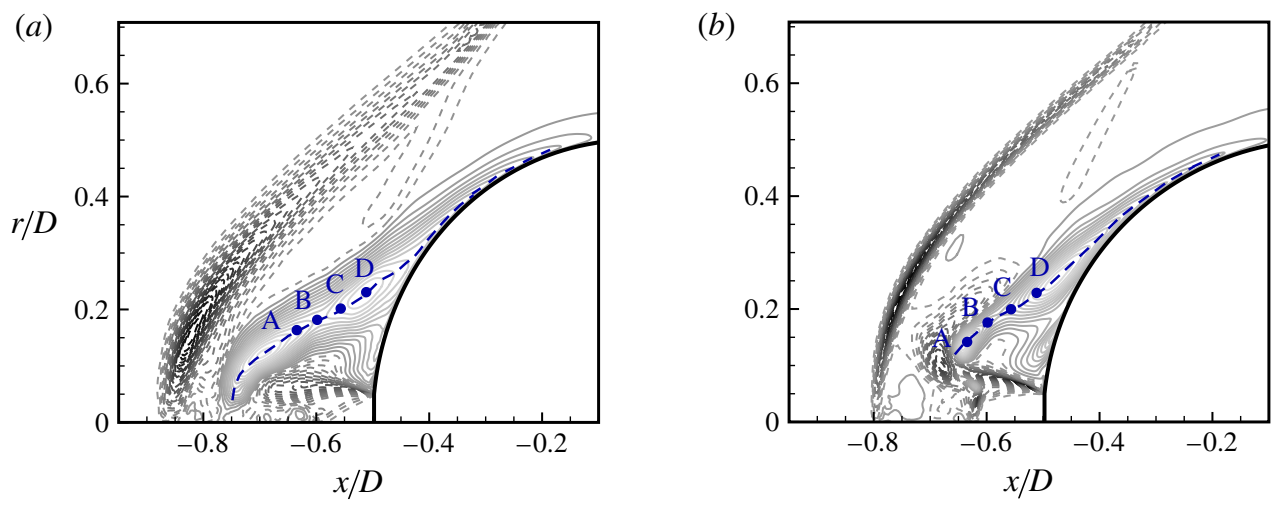

FIGURE 8. (Colour online) Distributions of the turbulent shear stress $\tau_{x r}^{\prime \prime}:(a) \mathscr{P}=0.816$ with the contour levels between $-0.035 U_{\infty}^{2}$ (dark, dashed lines) and $0.025 U_{\infty}^{2}$ (solid lines) and $(b) \mathscr{P}=1.633$ with the contour levels between $-0.028 U_{\infty}^{2}$ (dashed lines) and $0.018 U_{\infty}^{2}$ (bright, solid lines). Here, the solid bold, dashed lines with the four solid dots A-D, represent the locations of the local peak value of the shear stress and A-D denote the pressure probe locations.
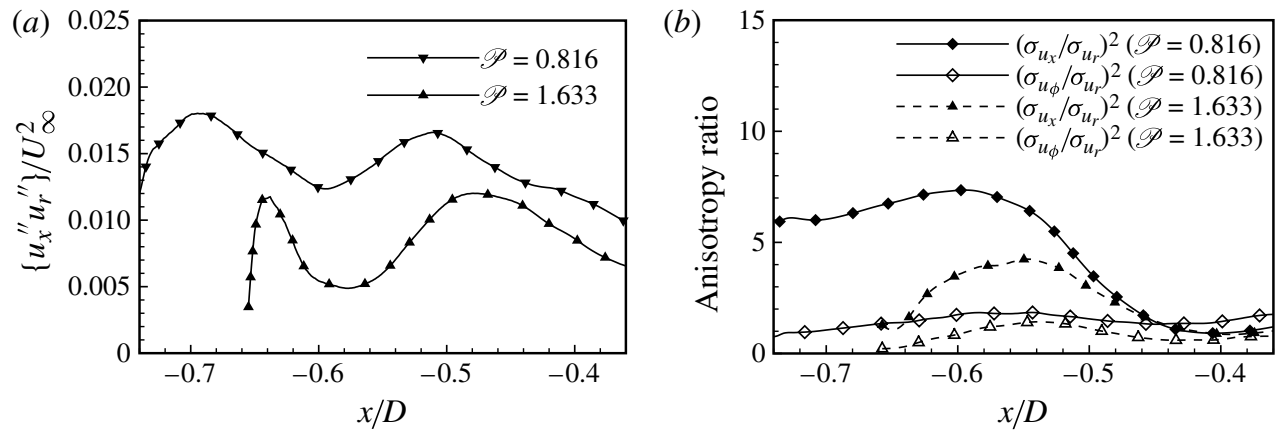

FIGURE 9. Distributions of the shear stress $(a)$ and the normal stress anisotropy ratios (b) along the shear layer.

in the conical shear-layer region and the boundary layer region after the shear-layer reattachment, while negative $\tau_{x r}^{\prime \prime}$ is distributed in the bow shock region and the jet layer region. For the purpose of better understanding the shear-layer evolution, the location of the shear layer in terms of local peak shear stress magnitude (Herrin \& Dutton 1997; Simon et al. 2007) is determined and exhibited by the dashed line with labels A-D in figure 8.

To reveal the development of the conical shear layer, the profiles of local peak Reynolds stresses $\left\{u_{i}^{\prime \prime} u_{j}^{\prime \prime}\right\} / U_{\infty}^{2}$ along the shear layer are shown in figure 9. The spatial evolution of the peak shear stress $\left\{u_{x}^{\prime \prime} u_{r}^{\prime \prime}\right\} / U_{\infty}^{2}$ is shown in figure $9(a)$. The distribution of this shear stress for the unstable state at $\mathscr{P}=0.816$ is higher than that for the stable state at $\mathscr{P}=1.633$ because the unstable state flow system is characterized by a stronger unsteadiness as mentioned above. Along the shear layer, the first maximum is related to the formation of vortex-ring-like coherent structures after the jet reverses its direction, which is similar to an incompressible counterflow (Yoda \& Fiedler 1996). The shear stress decreases as the coherent structures are 
distorted and convected downstream, and then increases rapidly to reach the second maximum which is associated with the onset of the recompression process due to the shear-layer reattachment. The shear stress decreases monotonically after approximately $x / D=-0.5$ because of the convex streamline curvature near the body surface (Debiève et al. 2003; Simon et al. 2007).

The Reynolds stress anisotropy in the shear layer is further analysed in terms of the ratios between the stresses which are expressed as $\sigma_{u_{i}}=\left\{u_{i}^{\prime \prime} u_{i}^{\prime \prime}\right\}^{1 / 2}$. Figure $9(b)$ shows the development of a primary-to-secondary stress ratio $\left(\sigma_{u_{x}} / \sigma_{u_{r}}\right)^{2}$ and a secondary-tosecondary stress ratio $\left(\sigma_{u_{\phi}} / \sigma_{u_{r}}\right)^{2}$ along the conical shear layer. It is observed that the streamwise normal stress is dominant for the two flow states. The profiles of $\left(\sigma_{u_{x}} / \sigma_{u_{r}}\right)^{2}$ vary smoothly for approximately $x / D<-0.55$ and then decay rapidly because of the reattachment process. Downstream of the reattachment, the streamwise fluctuations are damped to the same order as the radial ones, and the relative normal stress distributions seem to reach an equilibrium state in the boundary layer. Moreover, the secondary-to-secondary stress ratio $\left(\sigma_{u_{\phi}} / \sigma_{u_{r}}\right)^{2}$ remains around 1.0 , indicating little difference in the turbulent energy redistribution mechanism among these components.

The evolution of the primary-to-secondary stress ratio $\left(\sigma_{u_{x}} / \sigma_{u_{r}}\right)^{2}$ is associated with the flow motion feature (Amatucci et al. 1992; Herrin \& Dutton 1997; Simon et al. 2007). The value of $\left(\sigma_{u_{x}} / \sigma_{u_{r}}\right)^{2}$ lies in the range of 6.0-7.0 around the region of $x / D=-0.6$ for $\mathscr{P}=0.816$, which is related to the antisymmetric motion of the compressible shear layers behind a thick base (Amatucci et al. 1992). Correspondingly, $\left(\sigma_{u_{x}} / \sigma_{u_{r}}\right)^{2}$ is $\sim 3.5$ for $\mathscr{P}=1.633$ before its decay. For comparison, the primaryto-secondary stress ratio is around 3.0 in axisymmetric flows (Herrin \& Dutton 1997; Simon et al. 2007). The analysis suggests that the flow motion may have an antisymmetric feature for the unstable state and an axisymmetric one for the stable state.

\subsubsection{Proper orthogonal decomposition analysis of the velocity field}

The flow field can be quantitatively analysed using the proper orthogonal decomposition (POD) approach to extract energetic coherent structures from the simulation data (Berkooz et al. 1993). For a given flow property, $f(\boldsymbol{x}, t)$, the POD analysis can determine a set of orthogonal functions, $\phi_{j}(x), j=1,2, \ldots$, such that projection of $f$ onto the first $n$ functions

$$
\hat{f}(\boldsymbol{x}, t)=\bar{f}(\boldsymbol{x}, t)+\sum_{j=1}^{n} a_{j}(t) \phi_{j}(\boldsymbol{x}),
$$

has the smallest error, defined as $\left\langle\|f-\hat{f}\|^{2}\right\rangle_{t}$, where \langle\rangle$_{t}$ and $\|\cdot\|$ denote the time average and a norm in the $L^{2}$ space, respectively. Here, $a_{j}(t)$ represents the temporal variation of the $j$ th mode. The function $f$ can be extended to a vector $\boldsymbol{F}$ by introducing an appropriate inner product on $\boldsymbol{F}$. A more complete discussion of this subject can be found in Berkooz et al. (1993).

The analysis has been conducted using $N_{t}=1500$ snapshots of the streamwise velocity component, which is dominant among the three components, spanning a time period of $82.5 D / U_{\infty}$, and the temporal resolution is $0.055 D / U_{\infty}$, corresponding to a cutoff Strouhal number $\sim 9.09$. The energy of the $j$ th mode, $E_{j}$, is defined as

$$
E_{j}=\left\langle\left\|a_{j}(t) \phi_{j}(\boldsymbol{x})\right\|^{2}\right\rangle_{t}
$$


(a)

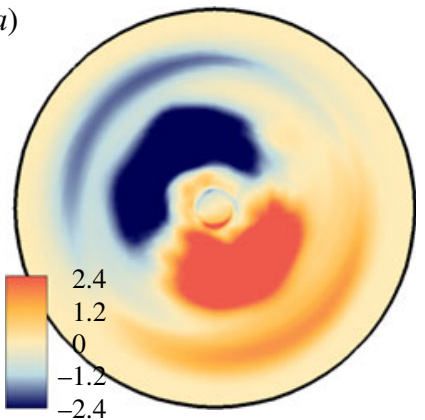

$(c)$

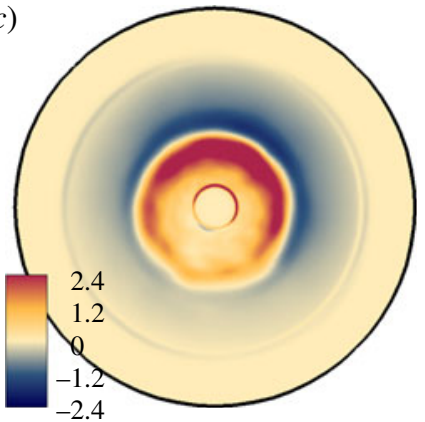

(b)

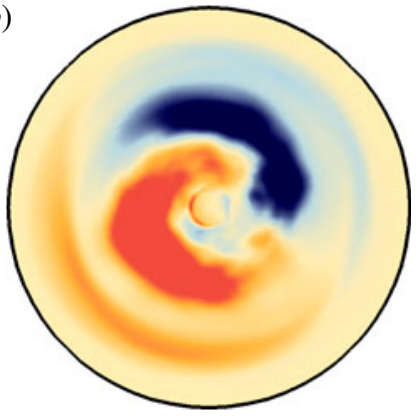

$(d)$

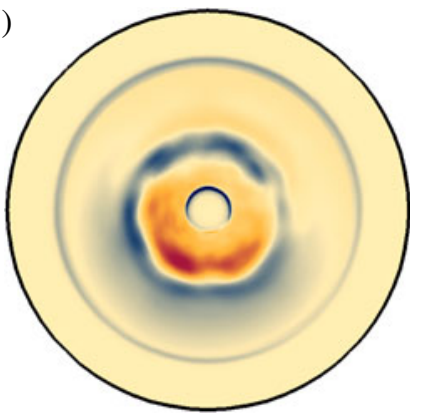

FIgURE 10. Spatial distributions of the first two POD modes: $(a)$ first mode and $(b)$ second mode for $\mathscr{P}=0.816$; $(c)$ first mode and $(d)$ second mode for $\mathscr{P}=1.633$.

(a)

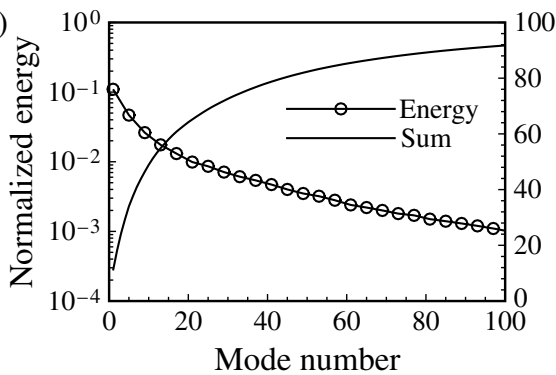

(b)

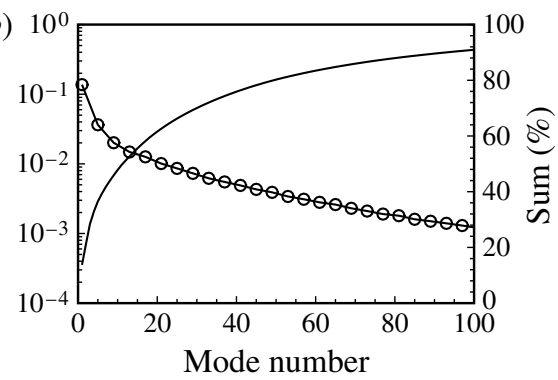

FIGURE 11. Energy distribution of POD modes based on the streamwise velocity for (a) $\mathscr{P}=0.816$ and $(b) 1.633$.

The normalized energy of the $m$ th mode is then defined as $E_{m} / \sum_{j=1}^{N_{t}} E_{j}$, and the energy sum from mode 1 to $m$ is solved as $\sum_{j=1}^{m} E_{j} / \sum_{j=1}^{N_{t}} E_{j}$. Using the time-varying coefficient $a_{j}(t)$ in (4.2), one can obtain the frequency spectrum of the $j$ th mode and the phase difference from the adjacent modes (Wang, Hsieh \& Yang 2005; Chen et al. $2010 b$ ). Figure 10 shows the mode shape distributions of the first two modes in the cross-section plane at $x / D=-0.55$ for $\mathscr{P}=0.816$ and 1.633 . Correspondingly, the energy distribution of the POD modes is shown in figure 11. The coherent structures can be captured by the POD analysis and presented by the mode shapes. 
For the unstable state at $\mathscr{P}=0.816$, the first two modes account for $\sim 18 \%$ of the total energy of the fluctuating velocity field as shown in figure 11(a) and exhibit antisymmetric shapes in figure $10(a, b)$. The captured energy is similar to the previous POD analysis for turbulent flows in turbomachinery (Wang et al. 2005) and turbulent jets in crossflow (Meyer et al. 2007). The dominant frequency of the first two modes is approximately $S t=0.25$ which is obtained by the spectral analysis of the time-varying coefficient $a_{j}(t)$ in (4.2). The dominant modes display the antisymmetric motion feature, which is associated with the off-axial flow motion in the cross-section plane.

For the stable state at $\mathscr{P}=1.633$, the first two modes occupy $\sim 21 \%$ of the total energy as shown in figure 11(b) and display axisymmetric shapes in figure $10(c, d)$. Similarly, from the time-varying coefficient $a_{j}(t)$ the dominant frequency of the two modes is determined as approximately $S t=1.51$. The mode shapes show that the dominant coherent structures have the axisymmetric feature. Thus, the analysis of the coherent structures has revealed the different dominant motions for the unstable and stable states, which are associated with the underlying mechanisms of the selfsustained motions and will be helpful in establishing the feedback models in $§$ 4.3.2.

\subsubsection{Statistical analysis of side loads}

The occurrence of turbulent flow structures influences unsteady loads, which can be obtained by integrating the unsteady pressure and friction on the body surface. To analyse the character of the fluctuating side loads, figure 12 shows a typical polar plot of the side loads, i.e. $F_{y}(t)$ and $F_{z}(t)$, which represent the $y$ - and $z$-direction components of the load in the cross-section plane, respectively. To qualify the statistics of the side loads for the axisymmetrical geometry considered here, we also plot a $95 \%$ confidence ellipse whose boundary in $\mathscr{R}^{2}$ space is defined by Deck \& Nguyen (2004):

$$
(\tilde{F}-M)^{t} C^{-1}(\tilde{F}-M)=2 \log (1-\alpha),
$$

where $\alpha=0.95, \tilde{F}=\left(F_{y}, F_{z}\right)^{t}$ denotes a bi-dimensional random variable defined by its mean value $M=\left[\bar{F}_{y}, \bar{F}_{z}\right]^{t}$ and its covariance $C=\overline{\tilde{F}} \tilde{F}^{t}-M M^{t}$. The number of points outside the equiprobability ellipse can then be compared with the theoretical number, $N(1-\alpha)$, corresponding to a two-dimensional Gaussian distribution (Deck \& Nguyen 2004). Moreover, if the covariance matrix is symmetric, the ellipse becomes a circle, which is centred at zero. As shown in figure 12 for $\mathscr{P}=0.816$ and 1.633 , we have identified that the calculated number of points outside the confidence circle is less than $0.1 \%$ of the theoretical number. Thus, we learn that, for an averaged axisymmetric flow, the mean side loads are absent, i.e. $\bar{F}_{y}=\bar{F}_{z}=0$.

To obtain quantitatively the statistical behaviour of the side loads, figure 13(a,b) shows the side-load probability density functions (p.d.f.s) for $\mathscr{P}=0.816$ and 1.633 , respectively. The computed results are close to a Rayleigh distribution with their discrepancies being less than $1 \%$. This feature is consistent with previous findings in different flows (e.g. Deck \& Nguyen 2004; Deck \& Thorigny 2007) and may be expected for an axisymmetric geometry because the Rayleigh distribution is the particular case of a $\chi^{2}$ distribution with two degrees of freedom, which correlates with normally distributed side-load components.

Further, figure 13(c,d) shows the p.d.f.s of the side-load direction denoted by the azimuthal angle $\phi$ with a normal distribution. It is seen that the computed p.d.f.s of the side-load direction fluctuate around the uniform law within the interval $[0,2 \pi]$ (or $\left[0^{\circ}, 360^{\circ}\right]$ ). The higher-order statistical quantities, such as the skewness factor $S_{\phi}$ and the kurtosis factor $K_{\phi}$ with their definitions, are given in table 2, which can 

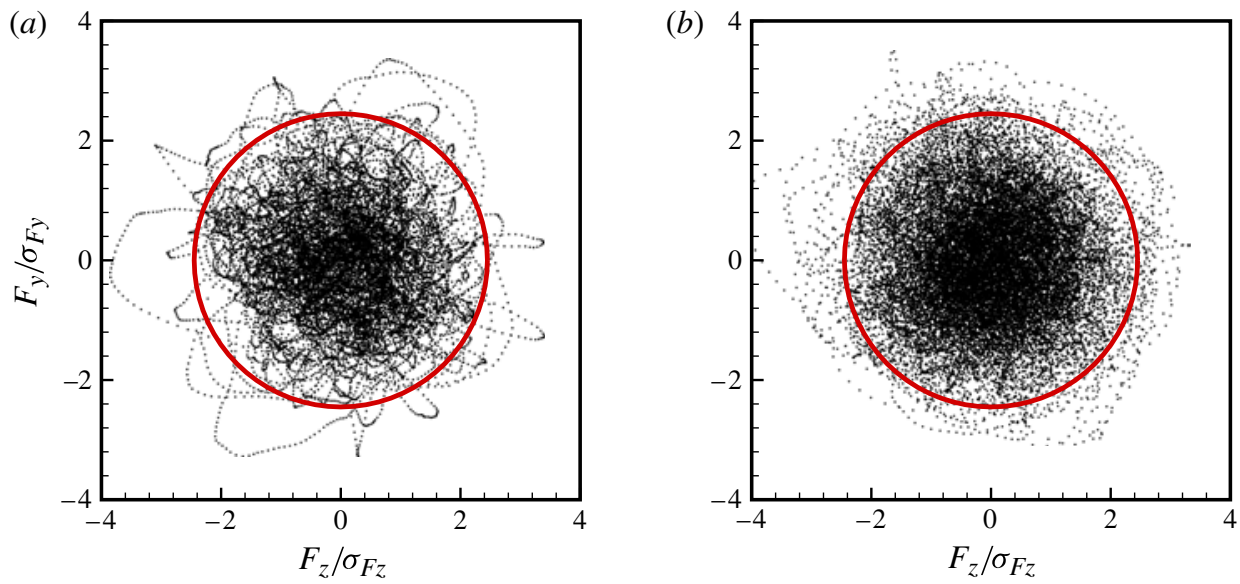

FIGURE 12. (Colour online) Polar plot of side loads and $95 \%$ confidence circle for (a) $\mathscr{P}=0.816$ and $(b) 1.633$.
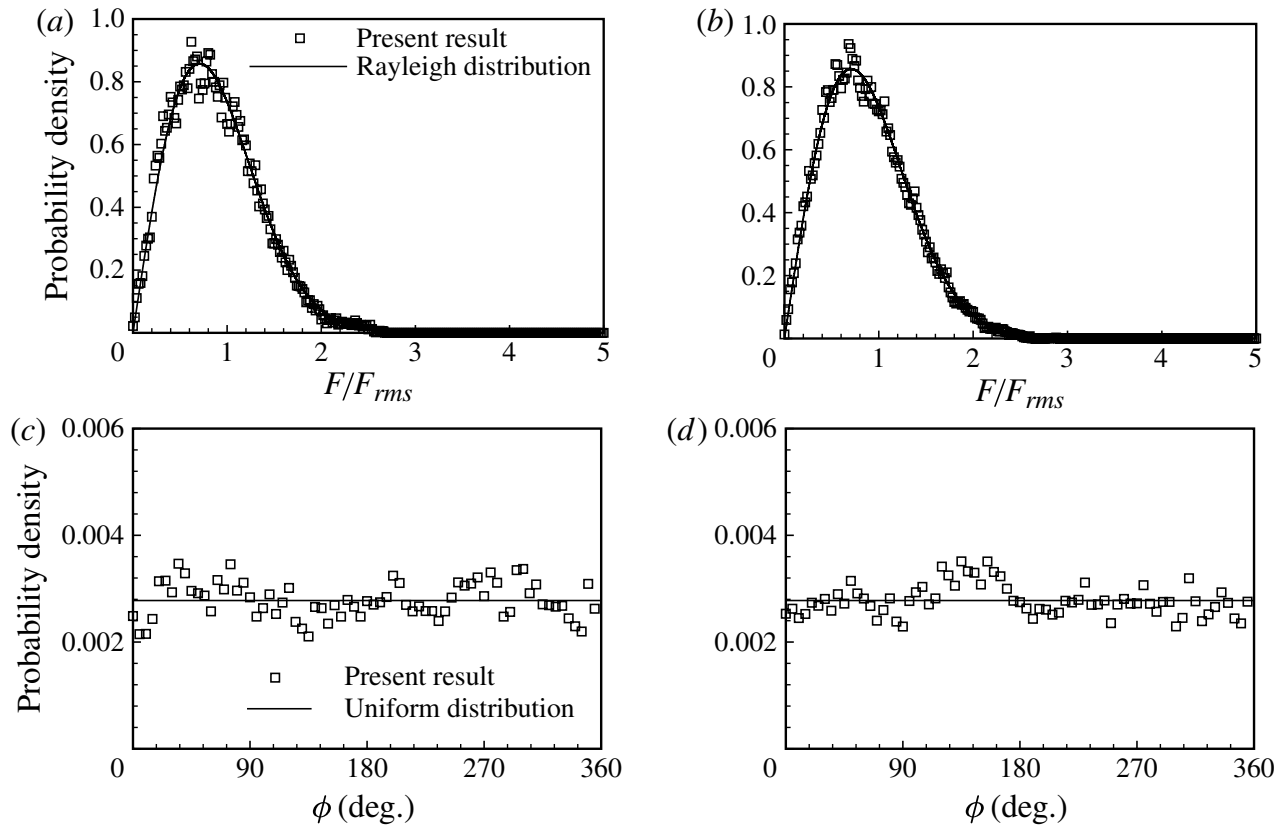

FIGURE 13. The p.d.f. of side-load amplitude for $(a) \mathscr{P}=0.816$ and $(b) 1.633$ as well as the side-load direction for $(c) \mathscr{P}=0.816$ and $(d) 1.633$.

then be compared with theoretical values of the uniform distribution. It is noted that the computed results agree with the theoretical values for the unstable $(\mathscr{P}=0.816)$ and stable $(\mathscr{P}=1.633)$ states. The skewness factor $S_{\phi}=0$ indicates the symmetry of the distribution, which means that no load direction is preferred. The kurtosis factor $K_{\phi}=1.8$ shows that the direction does not follow a Gaussian distribution (i.e. $K_{\phi}=3$ ). Thus, according to the statistical analysis, the side loads can be seen as a rotating vector uniformly distributed within the interval $[0,2 \pi]$ and whose magnitude 


$\begin{array}{lccccc}\text { Statistical moment } & \text { Uniform law } & \mathscr{P}=0.816 & \Delta(\%) & \mathscr{P}=1.633 & \Delta(\%) \\ \bar{\phi} / \pi & 1 & 0.999 & 0.1 & 0.992 & 0.8 \\ \sigma_{\phi}^{2} / \pi^{2} & 1 / 3 & 0.333 & 0.1 & 0.319 & 4.3 \\ S_{\phi}=\overline{\phi^{\prime 3}} /{\overline{\phi^{\prime 2}}}^{3 / 2} & 0 & 6.32 \times 10^{-3} & 0.6 & 0.027 & 2.7 \\ K_{\phi}={\overline{\phi^{\prime 4}}} /{\overline{\phi^{\prime 2}}}^{2} & 9 / 5 & 1.76 & 2.2 & 1.840 & 2.2\end{array}$

TABLE 2. Comparison of statistical moments between the present calculation and the uniform distribution.
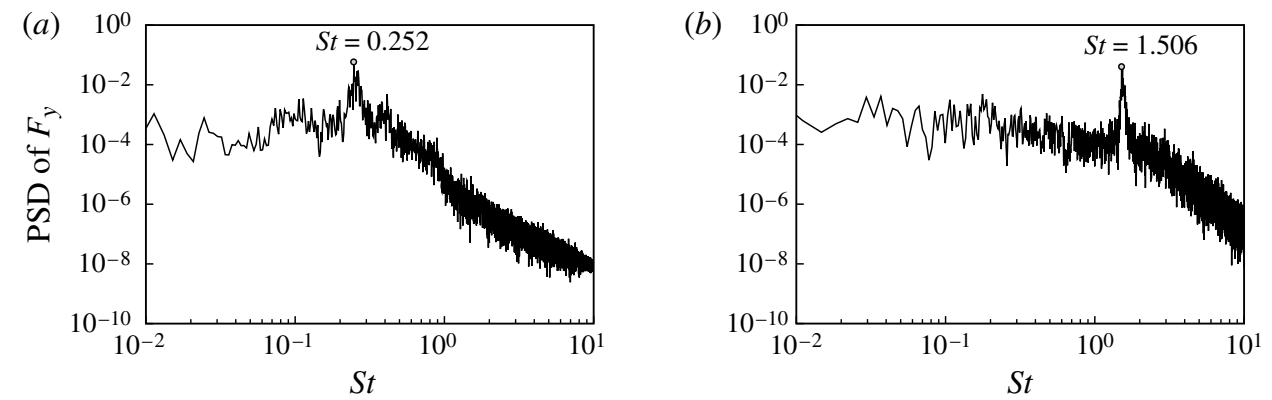

FIGURE 14. Spectrum of the load component $F_{y}$ for $(a) \mathscr{P}=0.816$ and $(b) 1.633$.

follows Rayleigh's law, consistent with the behaviour for an axisymmetric step flow (Deck \& Thorigny 2007).

\subsection{Spectral analysis and feedback models}

\subsubsection{Spectral analysis}

From the preceding description of the statistical behaviours of side loads, spectral analysis is further used to determine the dominant frequency of the flow evolution. Figure $14(a, b)$ shows the power spectral densities (PSDs) of $F_{y}(t)$ for $\mathscr{P}=0.816$ and 1.633 , respectively. The profiles of PSD contain a series of peaks including the highest one at $S t=0.252$ for the unstable state and $S t=1.506$ for the stable state, which are in agreement with the values predicted by the POD analysis. Moreover, based on the approach used by Deck \& Nguyen (2004), we can identify that the frequency range $S t \in[0.20,0.35]$ contributes $68 \%$ of the load component for the unstable state and the range $S t \in[1.40,1.65]$ contributes $50 \%$ of the load for the stable state.

Moreover, to examine the dominant frequency related to the recirculation region (figure 2) and the relevant spectrum behaviour, figure 15 shows the PSD of the time-dependent pressure in the boundary layer at the onset of the recirculation region. For the unstable state at $\mathscr{P}=0.816$, the PSD exhibits a dominant frequency around $S t=0.252$, as listed in table 1 , in agreement with the experimental data 0.262 or $f=3 \mathrm{kHz}$ (Fujita \& Karashima 1999; Fujita 2002). For the stable state at $\mathscr{P}=1.633$, the PSD indicates a dominant frequency $S t=1.506$, consistent with the experimental value 1.485 or $f=17 \mathrm{kHz}$ given in table 1 . Moreover, it is identified that the spectrum varies like $S t^{-4}$ at higher frequencies, which is caused by the 'active' inner-layer motion in the boundary layer (Bradshaw 1967) and is consistent with the findings in 
(a)

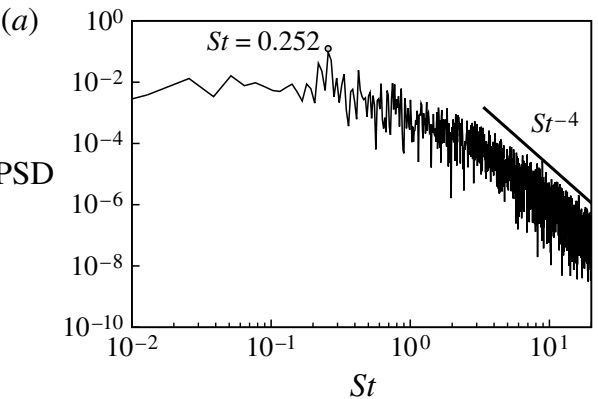

(b)

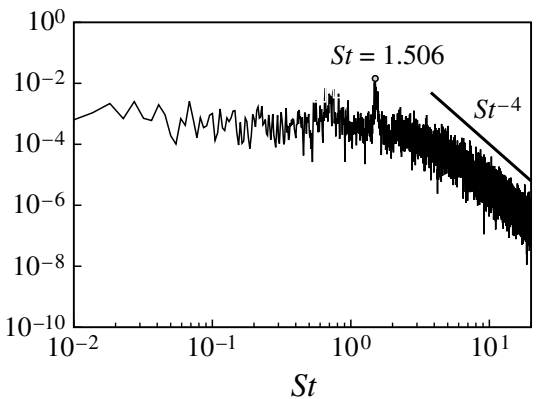

FIGURE 15. Spectrum of the pressure in the recirculation region for $(a) \mathscr{P}=0.816$ and $(b) 1.633$.

turbulent boundary layers (e.g. Simpson, Ghodbane \& McGrath 1987; Na \& Moin 1998).

The dominant frequency is closely related to the flow states and the coherent motions. From experimental observation and analysis, Fujita (2002) proposed that the lower frequency $f=3 \mathrm{kHz}$ in the unstable state may be induced by the unsteady off-axial motion of the flow system, and the higher frequency of $f=17 \mathrm{kHz}$ in the stable state may be caused by the axisymmetric motion. Thus, the above detailed analysis verifies Fujita's assumption. As shown in figure $10(a, b)$, we obtain that the dominant modes of the unstable state are antisymmetric with $S t=0.252$ (i.e. the dominant frequency of flow evolution detected in figures 14 and 15), which is related to the off-axial motion. Moreover, as exhibited in figure $10(c, d)$, the dominant modes of the stable state are axisymmetric with $S t=1.506$ (i.e. the dominant frequency of flow evolution), which is related to the axisymmetric coherent structure evolution.

\subsubsection{Feedback models}

Self-sustained motions of a jet from a blunt body opposing a supersonic flow have been observed (e.g. Finley 1966; Karashima \& Sato 1975; Shang et al. 2001). This feature is usually associated with shear-layer impingement phenomena and disturbance feedback (e.g. Rockwell \& Naudasher 1979; Shang et al. 2001; Fujita 2002). Based on injection experiments in high-speed flows, Shang et al. (2001) suggested a feedback mechanism whereby the oscillatory motion is sustained by the upstream disturbance propagation of selectively amplified frequencies from the free shear layer to the Mach disk through the embedded subsonic domain. Fujita (2002) also proposed a feedback model describing the evolution of the jet structure. However, they did not give the relevant feedback model to determine quantitatively the frequency. Here we will attempt to propose feedback models to predict the dominant frequencies in the unstable and stable states.

For the shear-layer impingement phenomena, a model was proposed by Tam (1974) based on acoustic resonance and it has been usually used to obtain the characteristic frequency of the oscillation:

$$
\frac{\mathscr{L}}{V_{d}}+\frac{\mathscr{L}}{a_{l o c}}=\frac{n}{f},
$$

where $\mathscr{L}$ represents the characteristic length over which the shear layer develops, $a_{l o c}$ is the local speed of sound and is associated with the upstream propagating disturbance, $V_{d}$ is the speed of the downstream propagating wave and $n$ is the stage of 

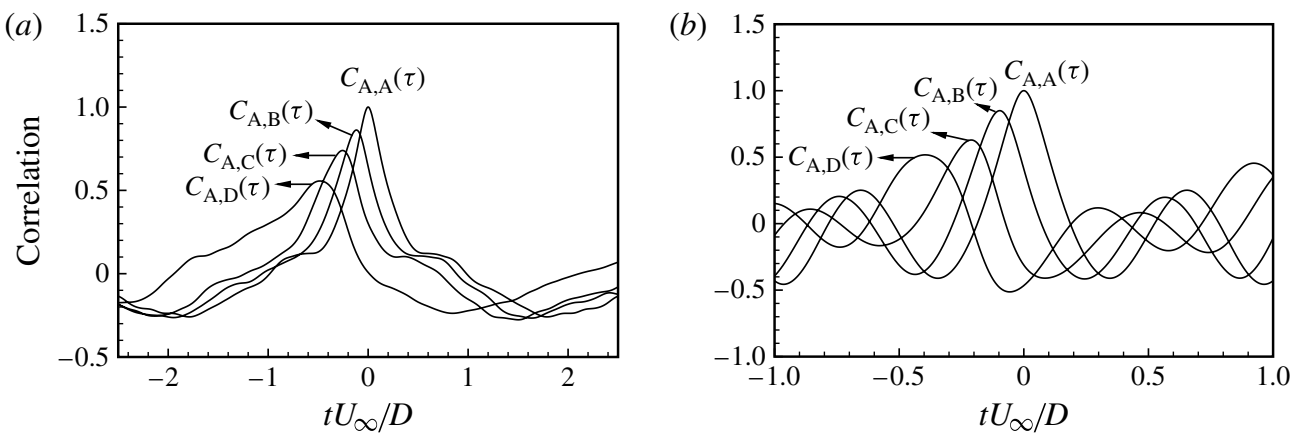

FIGURE 16. Cross-correlation of the downstream propagating pressure waves for (a) $\mathscr{P}=0.816$ and $(b) 1.633$.

the oscillation, such as vortex pairing, shear-layer oscillation, and so on (Ho \& Nosseir 1981; Deck \& Thorigny 2007).

Based on the above analysis, we propose a feedback loop of upstream propagating disturbance to the Mach disk through the recirculation subsonic region and downstream propagation in the conical shear layer. The characteristic length in (4.5) is reasonably well given by the distance, $L_{s}$, between the Mach disk and the jet injection point (Shang et al. 2001), and can be determined as $L_{s}=0.241 D$ for the unstable state and $L_{s}=0.126 D$ for the stable state from figure $2(a, b)$.

We here deal with the speed of downstream propagating pressure disturbances, which is used in the feedback model. Two-point cross-correlation of the unsteady pressure is used to quantitatively determine the propagation speed of the pressure disturbances along a given path. A covariance coefficient $C_{i j}$ for two pressure signals $p_{i}(t)$ and $p_{j}(t)$ with time delay $\tau$ can be defined as

$$
C_{i j}(\tau)=\frac{\left\langle\left(p_{i}(t)-\left\langle p_{i}\right\rangle_{t}\right)\left(p_{j}(t-\tau)-\left\langle p_{j}\right\rangle_{t}\right)\right\rangle_{t}}{\left\langle\left(p_{i}(t)-\left\langle p_{i}\right\rangle_{t}\right)^{2}\left(p_{j}(t)-\left\langle p_{j}\right\rangle_{t}\right)^{2}\right\rangle_{t}},
$$

where \langle\rangle$_{t}$ denotes time average. Then, the cross-correlation analysis is conducted for the probes A-D shown in figure 8 and the results are given in figure $16(a, b)$ for the unstable and stable state, respectively. Positive time delays are obtained, indicating that the pressure disturbances in the conical shear layer propagate downstream towards the body surface. The local propagation speed of the pressure disturbances can be calculated by dividing the streamwise spatial distances between neighbouring probes by the time delays between the peaks of the corresponding cross-correlations (Chen et al. 2010b). Then, the speed is obtained as approximately $0.30 U_{\infty}$ for the unstable state and $0.31 U_{\infty}$ for the stable state.

As the dominant motion exhibits axisymmetric shapes for the stable state, we can directly apply (4.5) to this case (Tam 1974). Based on the above description of the feedback loop, with a schematic in figure 17(a), the feedback model for stable flow state is simply proposed as

$$
T=\frac{L_{s}}{V_{d}}+\frac{L_{s}}{a_{l o c}} .
$$

Here, the speed of downstream propagation in the shear layer $V_{d}$ is $0.31 U_{\infty}$ and the local speed of sound in the recirculation region $a_{l o c}$ is obtained as $0.54 U_{\infty}$ for the 
(a)

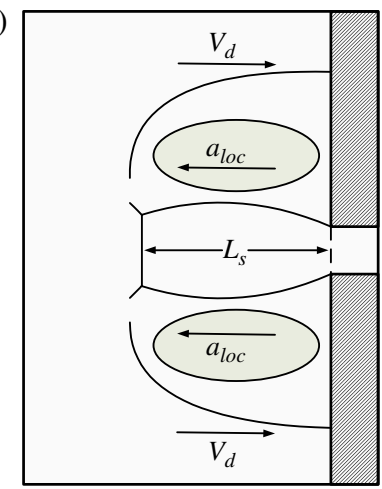

(b)

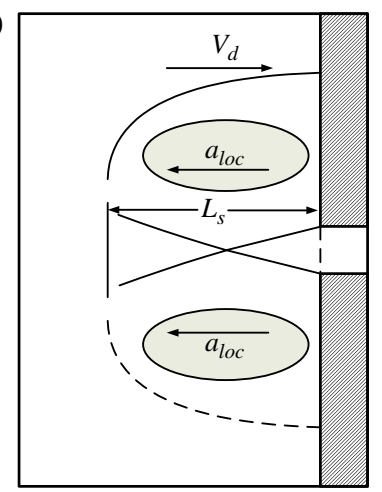

(c)

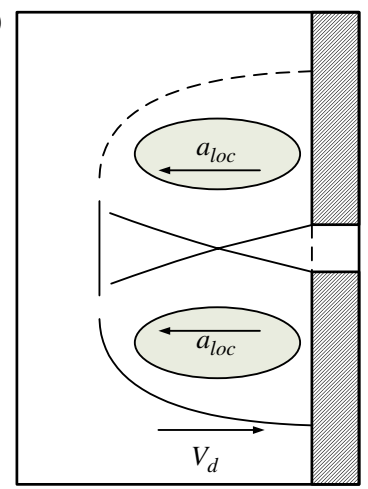

FIGURE 17. Schematics of feedback loops for the stable state $(a)$ and the unstable state $(b, c)$.

stable state. Then, the non-dimensional frequency of the feedback loop is calculated as $S t=1.563$, which is consistent with $S t=1.506$ as shown in figure $15(b)$.

Further, according to the analysis of the coherent structures of flow motion and the experimental findings (e.g. Fujita \& Karashima 1999; Fujita 2002), the dominant motion of the conical shear layer for the unstable state exhibits an antisymmetric character. Here, we will try to extend the above idea for the feedback model to the unstable state. Based on the analysis of flow evolution, it is noticed that the conical shear layer exhibits an off-axial flapping motion and the downstream-propagating waves occur alternately in one side and then the other side, as schematically exhibited in figure $17(b, c)$. Thus, we may refer to one complete period as the processes involved in both sides or as twice the period for the feedback loop in one side. We then assume that the mean speed over the complete period is taken as half the speed of downstream-propagating waves in one side, i.e. $\bar{V}_{d}=V_{d} / 2$. Based on the above analysis, a feedback model is proposed to describe one complete period of flow system as

$$
T=2\left(\frac{L_{s}}{\bar{V}_{d}}+\frac{L_{s}}{a_{l o c}}\right) .
$$

Here, $\bar{V}_{d}=0.15 U_{\infty}$ and $a_{l o c}=0.54 U_{\infty}$ for the unstable state. We apply (4.8) to obtain the non-dimensional frequency of the feedback loop $S t=0.244$, which is consistent with $S t=0.252$ obtained from the spectral analysis shown in figure 15(a). Moreover, note that a similar treatment has been applied to proposing the feedback model of a self-sustained shock motion on the upper and lower sides of a circular-arc aerofoil in transonic flow (Chen et al. 2010b).

Basically, we recognize the limitations of the simplified analysis of the feedback models; nevertheless, we feel that the results obtained from the models will be helpful in the understanding of the physical mechanisms of the self-sustained motions in the unstable and stable states.

\section{Concluding remarks}

Numerical investigation of a jet from a blunt body opposing a supersonic flow was carried out by means of an LES technique for two typical total pressure ratios of the jet to the free stream. Various fundamental mechanisms dictating the complex flow characteristics, including shock/jet interaction, shock/shear-layer interaction, turbulent 
shear-layer evolution and feedback mechanisms, were examined systematically and are summarized briefly as follows.

The flow features and structures depend mainly on the total pressure ratio $\mathscr{P}$. As the jet issues from the body against the supersonic flow, the bow shock stands away from the body surface and takes a form appropriate to the combined influence of the body and the jet flow. Based on the analysis of the mean and instantaneous flow behaviours, we have identified two typical flow states, i.e. the unstable and stable states, respectively. For the unstable state at $\mathscr{P}=0.816$, the oblique shocks generated in the jet column interact with regular reflections to form an X-type structure in the plane, and the bow shock interacting with the shear layer results in a self-sustained off-axis motion of flow structures. For the stable state at $\mathscr{P}=1.633$, the jet cell structure exhibits a Mach reflection with a well-established Mach disk, and the jet interacting with the Mach disk reverses its orientation as a conical shear layer convecting to the body surface. The existence of vortical structures in the flow field is also assessed. The small-scale vortical structures mainly occur in the jet column. As the jet terminates through the Mach disk and the fluid from the jet is deflected back, large-scale vortical structures develop gradually in the recirculation region.

The fluctuating quantities on the surface are examined. It is reasonably clearly identified that the pressure and temperature fluctuations for the unstable state are higher than those for the stable state. Moreover, the turbulent fluctuations are enhanced by the rapid deviation of the shear layer and the interaction with shock waves. The analysis of local peak Reynolds stresses along the conical shear layer indicates that the streamwise stress for the unstable state is higher than that for the stable state. The development of the primary-to-secondary stress ratio is associated with the flow motion feature and predicts that the antisymmetric motion is related to the unstable state and the axisymmetric motion to the stable state.

The coherent structures of the flow motion are analysed using the POD technique. The dominant modes for the unstable state exhibit antisymmetric patterns which are associated with the off-axial motion in the cross-section plane. Correspondingly, the dominant modes for the stable state display axisymmetric shapes which are related to the axisymmetric motion. Furthermore, the occurrence of coherent structures over the body surface influences unsteady loads. Statistical analysis of side loads indicates that they can be seen as a rotating vector uniformly distributed within the interval $[0,2 \pi]$ and whose magnitude follows Rayleigh's law for the unstable and stable states.

The feedback mechanism and dominant frequency of the self-sustained motions are further investigated. The dominant frequencies for the unstable and stable states are obtained based on the spectral analysis of the side load and the pressure signal in the flow field and agree well with the previous experimental data. It is confirmed that the lower frequency in the unstable state is induced by the unsteady off-axial motion of the flow system, and the higher frequency in the stable state is caused by the axisymmetric motion. Further, we clarify a feedback mechanism whereby the unsteady motion is sustained by the upstream-propagating disturbance to the Mach disk through the recirculation subsonic region and the downstream-propagating disturbance in the conical shear layer. Then, feedback models are proposed that reasonably well predict the dominant frequencies for the two flow states.

The authors are very grateful to Professor M. Fujita for the valuable discussions. This work was supported by the National Natural Science Foundation of China (Grant Nos 11072236 and 11132010) and the Foundation of the Chinese Academy of Sciences (Grant No. CXJJ-11-M69). 


\section{REFERENCES}

Amatucci, V. A., Dutton, J. C., Kuntz, D. W. \& Addy, A. L. 1992 Two stream, supersonic, wake flowfield behind a thick base. Part 1. General features. AIAA J. 30, 2039-2046.

Andreopoulos, Y., Agui, J. H. \& Briassulis, G. 2000 Shock wave-turbulence interactions. Annu. Rev. Fluid Mech. 32, 309-345.

Berkooz, G., Holmes, P. \& Lumley, J. L. 1993 The proper orthogonal decomposition in the analysis of turbulent flows. Annu. Rev. Fluid Mech. 25, 539-575.

Bodony, D. J. \& LELE, S. K. 2005 On using large-eddy simulation for the prediction of noise from cold and heated turbulent jets. Phys. Fluids 17, 085103.

Bogey, C. \& BAILly, C. 2006 Large eddy simulations of transitional round jets: influence of the Reynolds number on flow development and energy dissipation. Phys. Fluids 18, 065101.

Bourdon, C. J. \& Dutton, J. C. 2000 Shear layer flapping and interface convolution in a separated supersonic flow. AIAA J. 38, 1907-1915.

BRADSHAW, P. 1967 The turbulence structure of equilibrium boundary layers. J. Fluid Mech. 29, 625-645.

BRADShaw, P. 1974 The effect of mean compression or dilatation on the turbulence structure for supersonic boundary layers. J. Fluid Mech. 63, 449-464.

Chen, L.-W., XU, C.-Y. \& LU, X.-Y. $2010 a$ Large eddy simulation of opposing-jet-perturbed supersonic flow past a hemispherical noses. Mod. Phys. Lett. B 24, 1287-1290.

Chen, L.-W., XU, C.-Y. \& LU, X.-Y. $2010 b$ Numerical investigation of the compressible flow past an aerofoil. J. Fluid Mech. 643, 97-126.

Debiève, J.-F., ARdissone, J.-P. \& Dussauge, J.-P. 2003 Shock motion and state of turbulence in a perturbed supersonic flow around a sphere. J. Turbul. 4, 1-15.

DeCK, S. \& NGUYEn, A. T. 2004 Unsteady side loads in a thrust-optimized contour nozzle at hysteresis regime. AIAA J. 42, 1878-1888.

DECK, S. \& THORIGNy, P. 2007 Unsteadiness of an axisymmetric separating-reattaching flow: numerical investigation. Phys. Fluids 19, 065103.

Ferrante, A., Matheou, G. \& Dimotakis, P. E. 2011 LES of an inclined sonic jet into a turbulent crossflow at Mach 3.6. J. Turbul. 12, 1-32.

FINLEY, P. J. 1966 The flow of a jet from a body opposing a supersonic free stream. J. Fluid Mech. 26, 337-368.

Freund, J. B., Lele, S. K. \& Moin, P. 2000 Compressibility effects in a turbulent annular mixing layer. Part 1. Turbulence and growth rate. J. Fluid Mech. 421, 229-267.

FUJiTA, M. 1995 Axisymmetric oscillations of an opposing jet from a hemispherical nose. AIAA J. 33, 1850-1856.

Fujita, M. 2002 Three-dimensional oscillations of a supersonic opposing jet flow around a hemispherical nose. J. Japan Soc. Aeronaut. Space Sci. 50, 373-379.

FujitA, M. \& KARAshima, K. 1999 An experimental and computational study on self-excited oscillations in supersonic opposing jet flow. Trans. Japan Soc. Aeronaut. Space Sci. 42, $112-119$.

Garnier, E., Sagaut, P. \& Deville, M. 2002 Large eddy simulation of shock/homogeneous turbulence interaction. Comput. Fluids 31, 245-268.

GÉnin, F. \& Menon, S. 2010 Dynamics of sonic jet injection into supersonic crossflow. J. Turbul. 11, $1-30$.

Georgiadis, N. J., Rizzetta, D. P. \& Fureby, C. 2010 Large-eddy simulation: current capabilities, recommended practices, and future research. AIAA J. 48, 1772-1784.

Herrin, J. L. \& DutTon, J. C. 1997 The turbulence structure of a reattaching axisymmetric compressible free shear layer. Phys. Fluids 9, 3502-3512.

Hill, D. J., Pantano, C. \& Pullin, D. I. 2006 Large-eddy simulation and multiscale modelling of a Richtmyer-Meshkov instability with reshock. J. Fluid Mech. 557, $29-61$.

Ho, C. M. \& Nosseir, N. S. 1981 Dynamics of an impinging jet. Part 1. The feedback phenomenon. J. Fluid Mech. 105, 119-142. 
Hudy, L. M., NAguiB, A. M. \& Humphreys, W. M. 2003 Wall-pressure-array measurements beneath a separating/reattaching flow region. Phys. Fluids 15, 706-717.

Jeong, J. \& Hussain, F. 1995 On the identification of a vortex. J. Fluid Mech. 285, 69-94.

Karashima, K. \& SATO, K. 1975 An experimental study of an opposing jet. Bull. Inst. Space Aeronaut. Sci. Univ. Tokyo 11, 53-64.

KaWAI, S. \& FUJII, K. 2005 Computational study of supersonic base flow using hybrid turbulence methodology. AIAA J. 43, 1265-1275.

Lu, X.-Y., WAng, S.-W., Sung, H. G., Hsieh, S. Y. \& YANG, V. 2005 Large-eddy simulations of turbulent swirling flows injected into a dump chamber. J. Fluid Mech. 527, 171-195.

LuMLEY, J. L. 1967 Rational approach to relations between motions of differing scales in turbulent flows. Phys. Fluids 10, 1405-1408.

Matheou, G., Bonanos, A. M., Pantano, C. \& Dimotakis, P. E. 2010 Large-eddy simulation of mixing in a recirculating shear flow. J. Fluid Mech. 646, 375-414.

Meyer, K. E., Pedersen, J. M. \& ÖzCan, O. 2007 A turbulent jet in crossflow analysed with proper orthogonal decomposition. J. Fluid Mech. 583, 199-227.

NA, Y. \& MoIN, P. 1998 The structure of wall-pressure fluctuations in turbulent boundary layers with adverse pressure gradient and separation. J. Fluid Mech. 377, 347-373.

Piomelli, U. 1999 Large-eddy simulation: achievements and challenges. Prog. Aerosp. Sci. 35, 335-362.

Pope, S. B. 2000 Turbulent Flows. Cambridge University Press.

Pope, S. B. 2004 Ten questions concerning the large-eddy simulation of turbulent flows. New J. Phys. 6, 35.

Rai, M. M. \& Chakravarthy, S. 1993 Conservative high-order accurate finite difference method for curvilinear grids. AIAA Paper 93-3380.

RIBNER, H. S. 1954 Shock-turbulence interaction and the generation of noise. NACA Tech. Note 3255 .

RoBINET, J.-C. 2007 Bifurcations in shock wave/laminar boundary layer interaction: global instability approach. J. Fluid Mech. 579, 85-112.

Robinson, S. K. 1991 Coherent motions in the turbulent boundary layers. Annu. Rev. Fluid Mech. 23, 601-639.

Rockwell, D. \& Naudasher, E. 1979 Self-sustained oscillations of the impinging free shear layers. Annu. Rev. Fluid Mech. 11, 67-94.

SHANG, J. S. 2002 Plasma injection for hypersonic blunt-body drag reduction. AIAA J. 40, $1178-1186$.

Shang, J. S., Hayes, J., Wurtzler, K. \& Strang, W. 2001 Jet-spike bifurcation in high-speed flows. AIAA J. 39, 1159-1165.

Simon, F., Deck, S., Guillen, P., Sagaut, P. \& Merlen, A. 2007 Numerical simulation of the compressible mixing layer past an axisymmetric trailing edge. J. Fluid Mech. 591, 215-253.

Simpson, R. L., Ghodbane, M. \& MCGrath, B. E. 1987 Surface pressure fluctuations in a separating turbulent boundary layer. J. Fluid Mech. 177, 167-186.

TAM, C. K. W. 1974 Discrete tones of isolated airfoils. J. Acoust. Soc. Am. 55, 1173-1177.

Thomas, J. L. \& SAlAS, M. D. 1986 Far-field boundary conditions for transonic lifting solutions to the Euler equations. AIAA J. 24, 1074-1080.

Viti, V., Neel, R. \& Schetz, J. A. 2009 Detailed flow physics of the supersonic jet interaction flow field. Phys. Fluids 21, 046101.

WANG, S. W., Hsieh, S. Y. \& YANG, V. 2005 Unsteady flow evolution in swirl injector with radial entry. I. Stationary conditions. Phys. Fluids 17, 045106.

WARREN, C. H. E. 1950 An experimental investigation of the effect of ejecting a coolant gas at the nose of a bluff body. J. Fluid Mech. 8, 400-417.

WU, J.-Z., LU, X.-Y. \& ZHUANG, L.-X. 2007 Integral force acting on a body due to local flow structures. J. Fluid Mech. 576, 265-286. 
XU, C.-Y., CHEN, L.-W. \& LU, X.-Y. 2009 Numerical simulation of shock wave and turbulence interaction over a circular cylinder. Mod. Phys. Lett. B 23, 233-236.

Xu, C.-Y., Chen, L.-W. \& LU, X.-Y. 2010 Large-eddy simulation of the compressible flow past a wavy cylinder. J. Fluid Mech. 665, 238-273.

YodA, M. \& FiEdLER, H. E. 1996 The round jet in a uniform counterflow: flow visualization and mean concentration measurements. Exp. Fluids 21, 427-436. 